UCRL-ID--108262

DE92 006638

\title{
Mapping Acoustic Emissions from Hydraulic Fracture Treatments Using Coherent Array Processing: Concept
}

\author{
David B. Harris \\ Robert J. Sherwood \\ Stephen P. Jarpe \\ Philip E. Harben \\ Lawrence Livermore National Laboratory
}

September, 1991

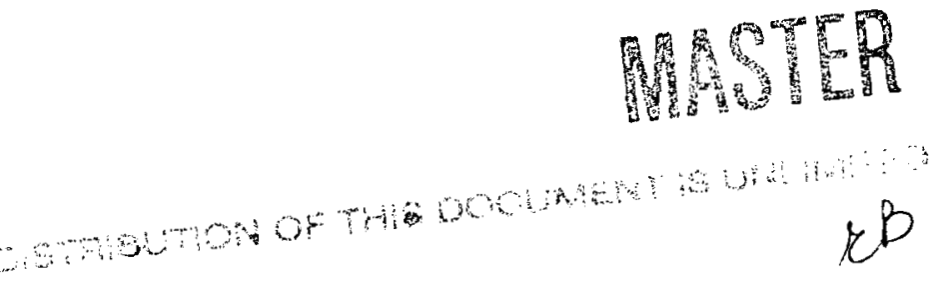




\section{DISCLAIMER}

This report was prepared as an account of work sponsored by an agency of the United States Government. Neither the United States Government nor any agency Thereof, nor any of their employees, makes any warranty, express or implied, or assumes any legal liability or responsibility for the accuracy, completeness, or usefulness of any information, apparatus, product, or process disclosed, or represents that its use would not infringe privately owned rights. Reference herein to any specific commercial product, process, or service by trade name, trademark, manufacturer, or otherwise does not necessarily constitute or imply its endorsement, recommendation, or favoring by the United States Government or any agency thereof. The views and opinions of authors expressed herein do not necessarily state or reflect those of the United States Government or any agency thereof. 


\section{DISCLAIMER}

Portions of this document may be illegible in electronic image products. Images are produced from the best available original document. 


\section{Mapping Acoustic Emissions from Hydraulic Fracture Treatments Using Coherent Array Processing: Concept}

\subsection{Introduction}

\subsection{Background}

Hydraulic fracturing is a widely-used well completion technique for enhancing the recovery of gas and oil in low-permeability formations. Hydraulic fracturing consists of pumping fluids into a well under high pressure (1000 - $5000 \mathrm{psi})$ to wedge-open and extend a fracture into the producing formation. The fracture acts as a conduit for gas and oil to flow back to the well, significantly increasing communication with larger volumes of the producing formation. The method has been employed since 1949, and more than 800,000 treatments had been completed prior to 1981 [Waters, 1981]. As of that time, 35 to 40 percent of all wells drilled in the U.S. were hydraulically fractured. The percentage is likely to increase as more marginal fields are exploited. As of 1981, 25 to 30 percent of proven domestic oil and gas reserves were made economically recoverable by hydraulic fracturing.

Hydraulic fracturing is very expensive, but cost effective. In the central California diatomite fields, typical treatment costs exceed $\$ 100,000$ per well. Costs up to $\$ 750,000$ per well are known [Waters, 1981]. In spite of the expense, hydraulic fracturing is often a profitable treatment. For example, in the central California fields, much of the oil reserve is locked up in diatomites, which are simultaneously highly porous and highly impermeable. In the last 80 years, less than $10 \%$ of the estimated reserve has been produced in some fields there. Since hydraulic fracturing began in the mid 1980's, production there has doubled or tripled, justifying a $\$ 75$ million investment in the process in 1991 [Chevron World, 1991].

Because the method is so expensive, it is used judiciously. Treatments of individual wells, and of whole reservoirs are carefully planned, simulated and monitored [Veatch, 1983a; Veatch, 1983b; Cleary, 1988; Anderson and Phillips, 1988; Economides, 1987; Elbel, 1988; Acharya, 1988]. Reservoir engineers need information on the geometry and size of fractures to plan successive stages of fracturing operations in individual wells, and to plan the spacing of fractured wells in a field. Most fractures have vertically oriented planar structures (Fig. 1). Optimum design of an individual fracture treatment in a well is a tradeoff between the expected production return from a given fracture volume (usually parameterized by fracture length, assuming a constant height and thickness) and the cost of extending the fracture. Costs increase rapidly with increasing fracture length, resulting in optimum lengths of several hundred to a few thousand feet. The density of fractured wells is a primary consideration in the exploitation of entire reservoirs. Fractures are designed 


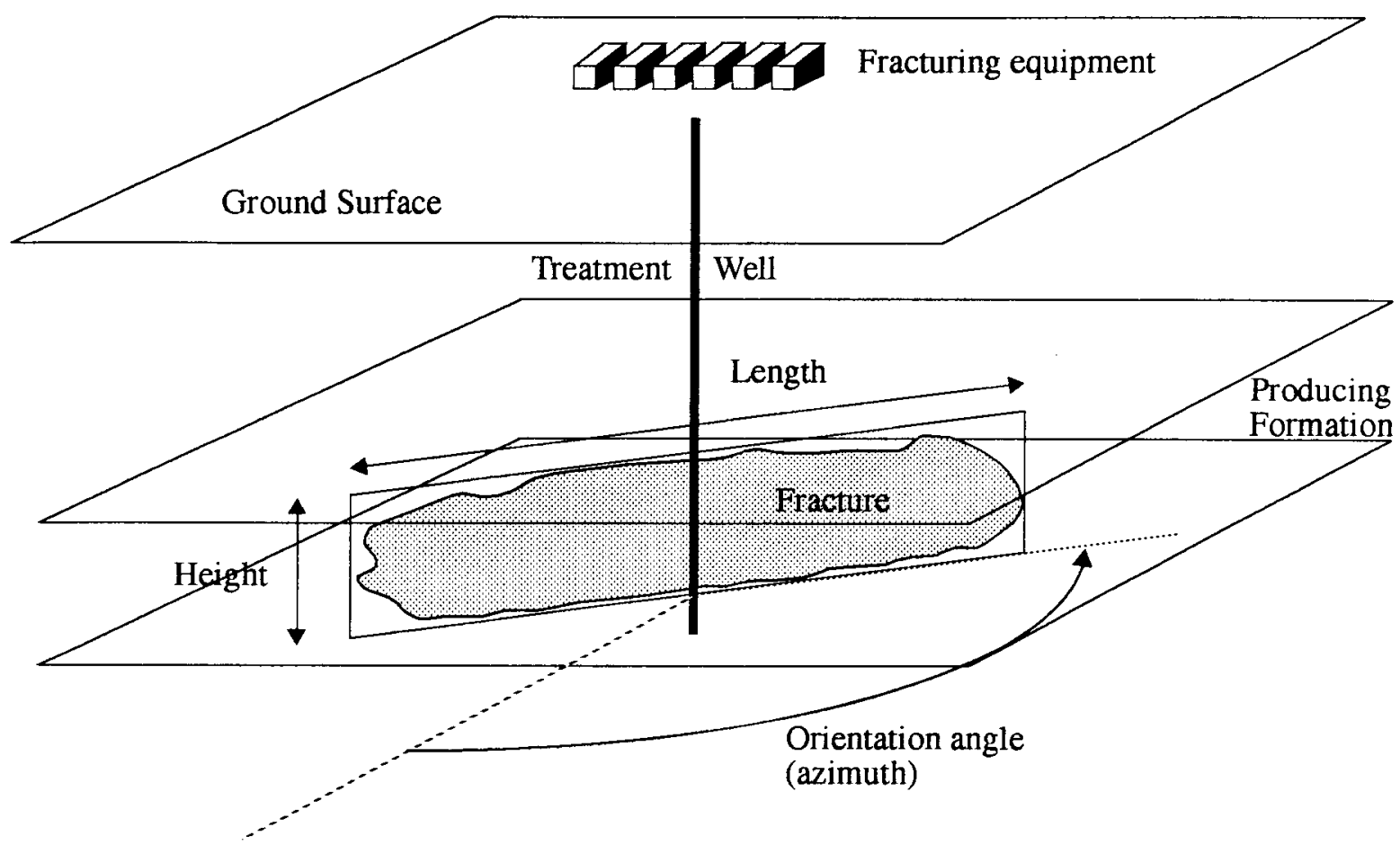

Figure 1 A typical fracture has a vertically-oriented planar structure dictated by the principal directions of stress in the medium. At depths greater than one to two thousand feet, the lithostatic (overburden) pressure prevents horizontal planar fractures from developing. A fracture's geometry is described in terms of its height, length, and azimuth. The fracture orientation is usually parallel to the principal axis of stress in the medium, since the face of the fracture is opened against the direction of least stress. The fracture may be confined vertically by layers of higher-strength rock above and below the injection point. A fracture is usually considered to grow outward symmetrically about the borehole, developing two "wings". The wing length is roughly half the overall fracture length.

not to intersect, since intersections would result in one well "stealing" the production of another. Such "fratricide" among wells is highly uneconomical. An optimal well spacing efficiently produces a formation by packing the fractures as densely as possible without overlap.

A typical fracturing treatment consists of several stages. The first is perforation. Wells are typically cased with steel tubing. To provide communication with the formation to be fractured, a perforation gun containing shaped charges is lowered to the treatment horizon. The charges are detonated, burning holes through the casing and several feet into the formation. A portion of the casing up to several hundred feet in extent may be perforated. Following perforation, the well below the perforations is isolated by insertion of an expandable plug (a bridge plug). The well is pressurized by pumps at the surface to the point where fluids exiting the perforations open a fracture. As pumping progresses, the fracture extends into the formation, perhaps hundreds of feet from the well bore. When the fracture is judged to be fully extended, sand is added to the slurry to prop open the fracture when 
the pressure is withdrawn. Usually a gel agent is added to the slurry to increase the viscosity of the fracturing fluid. Higher viscosity improves transport of the proppant (sand) into the extremities of the fracture.

Wells in fields with several producing horizons may be fractured in stages at multiple depths. The pause between stages required for perforation at the next horizon and resetting the bridge plug provides engineers with an opportunity to assess performance of the justcompleted stage, and make adjustments as necessary for the next treatment stage. This time interval may be a few hours in duration to overnight in treatments lasting several days. A commercially viable diagnostic would provide information on the lateral extent, orientation and height of fractures during this period.

From an operational standpoint, arguments can be made for the desirability of diagnostics that are available both much more quickly and on longer time scales. Real-time diagnostics would be especially valuable, if feasible, because they could be used to guide treatment dynamically [Cleary, 1988]. Often decisions to change such treatment parameters as fluid pressure, proppant load and gel viscosity have to be made as the treatment is underway. In principle, real-time diagnostics could minimize the cost to achieve a desired fracture size and geometry. In addition, an argument can be made for diagnostics that would provide detailed information on fracture geometry within a few days or even weeks. A diagnostic of this sort could be used more sparingly to plan treatment of an entire field. Information about the orientation and extent of one fracture might be taken to apply to other nearby wells, providing insight on the necessary spacing of wells for optimal reservoir exploitation.

The need for speed is not the only constraint on commercially viable diagnostics. Cost is perhaps even more important. Reservoir engineers with whom we have spoken insist that diagnostics should not consume more than $10 \%$ of their treatment budget. In the central California fields, that requirement translates to a $\$ 10,000$ to $\$ 20,000$ budget per treatment for monitoring operations.

\subsection{Existing Diagnostics}

A wide variety of diagnostic methods have been attempted and are used for estimating the distribution of hydraulically induced fractures. In this discussion, we will divide them into non-acoustic and acoustic methods. As our proposed method is acoustic (more accurately, seismic), we will concentrate on acoustic methods.

We first describe the non-acoustic alternatives briefly. As of 1983, post-fracturing temperature-decay profile (PFTDP) logs were the most common technique for estimating fracture height at the well-bore [Veatch, 1983b]. The presence of fracturing fluids alters the temperature of the formation in the vicinity of the well-bore, a disturbance that can be sensed by a tool that logs temperature continuously in the well past the fracture horizon. Radioactive doppants are sometimes added to the fracturing fluids. The distribution of these doppants can be logged in a similar manner. A variety of other logs such as the tube wave reflection log (TWRL), the cement evaluation log (CEL), and the variable density $\log$ (VDL) respond to fractures in the vicinity of the well [Hunt, 1991]. All of these diag- 
nostics are characterized by shallow penetration into the formation. Consequently, they measure fracture height only in the immediate vicinity of the wellbore. Although these methods offer only limited fracture characterization, they provide timely information immediately following the pumping operation.

Another commercially viable technique uses tiltmeters on the ground surface around the treatment well to delineate the azimuth and horizontal extent of the fracture. Though widely used, this method's resolution is poor for fracture depths greater than 5,000 feet [Mauk and Mahrer, 1988].

Perhaps the most commonly used real-time diagnostics are model-based methods that make inferences on fracture length and height from dynamic measurements of the pumping pressure, sometimes downhole pressure, and post-fracture pressure decline [Veatch, 1983b]. These methods are inexpensive and permit real-time control of the treatment parameters. However, they are indirect, relying upon models of fracture growth that may not capture the complete behavior of a heterogeneous medium.

Finally, an effective calibration of fracture volume is available from the amount of sand that is pumped into the well as proppant.

A considerable amount of research has been conducted on the use of acoustic (microseismic) emissions to delineate fracture growth. Acoustic emissions (discrete microseismic events) from hydraulic fractures were identifed as early as 1975 [Power, 1977; Shuck and Keech, 1975]. The use of transient signals to map the location of discrete sites of emission along fractures has been the focus of most research on methods for delineating fractures [Fehler, et al., 1987; Thorne and Morris, 1988a; Thorne and Morris 1988b; Fix et al., 1989; Fehler, 1989]. These methods depend upon timing the arrival of compressional (P) or shear (S) waves from discrete fracturing events at one or more clamped geophones in the treatment well or in adjacent monitoring wells. Using a propagation model, the arrival times are used to estimate the distance from each sensor to the fracturing event. If more than one sensor is available, the event location can be triangulated (Fig. 2). If a single three-component sensor is used, the direction to the event (and, hence, the location) can be estimated from the principal axis of polarization. In addition, long-period seismometers on the ground surface have detected signals from fracturing events. These detections have not resulted in reliable fracture locations [Mauk and Mahrer, 1988].

Discrete microseismic methods hold considerable promise for delineating the entire fracture distribution. Hence, they are the best available methods for complete fracture characterization. Their principal disadvantages are that they are slow, expensive and require high signal-to-noise ratio (SNR) recordings. They are slow because the individual transient seismic signals that are automatically detected must be manually screened from noise bursts. The signals with sufficiently high SNR must be selected, and the separate P and S arrivals manually timed. This procedure must be carried out repetitively for a sufficiently large number of events (hundreds) that the resulting event locations clearly delineate the fracture. The process is time consuming (and, thus, expensive), taking at least a month of effort with trained analysts for a single fracturing treatment [Thorne and Morris, 1988a]. The signals from fracture-induced microevents must have a sufficiently high SNR that 


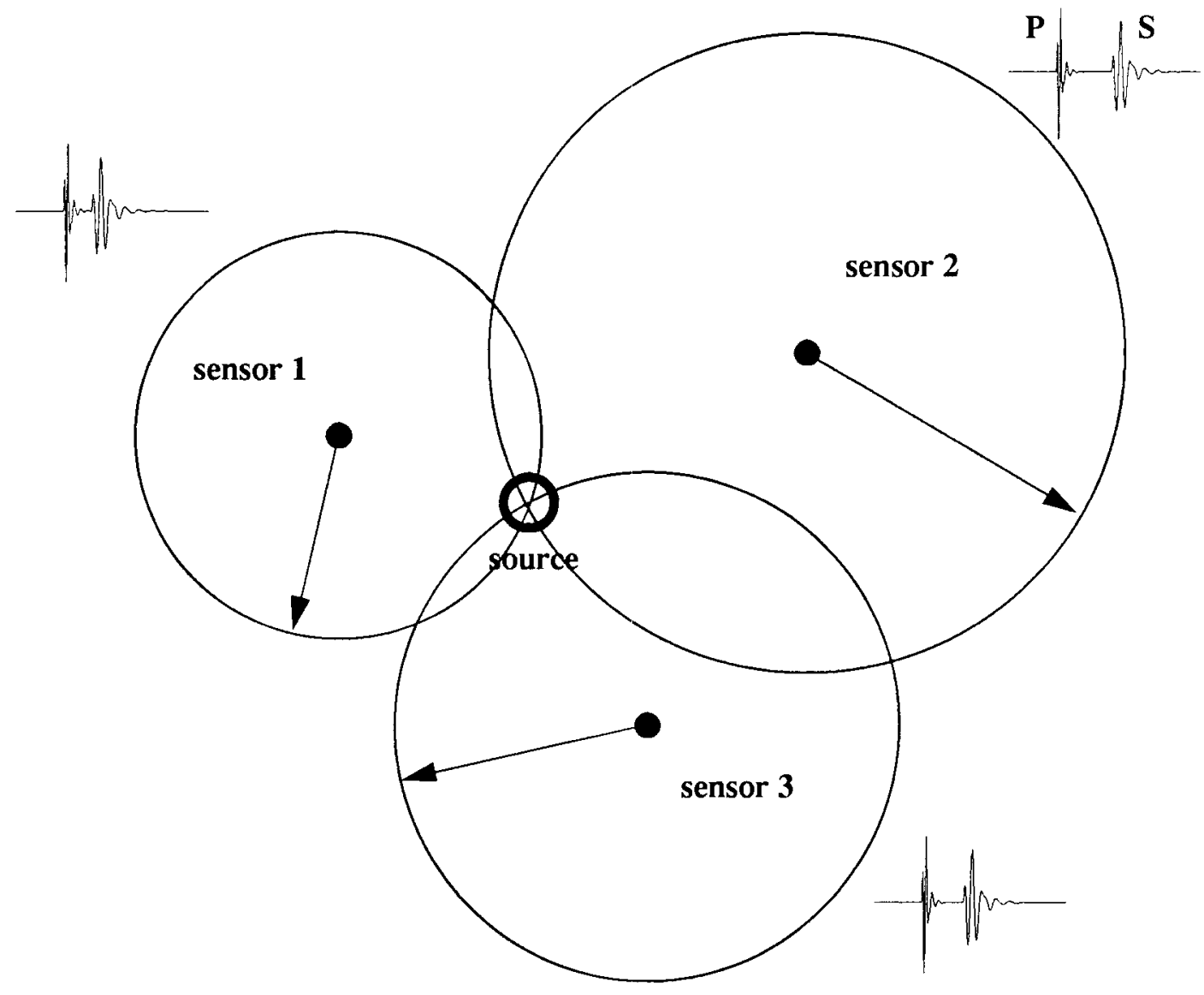

Figure 2 Transient signal methods triangulate hundreds of microseismic event locations to delineate a fracture system. Compressional (P) and shear (S) waves are detected, identified and the onsets of their arrivals are timed. Using a velocity model, and the fact that $P$ and $S$ waves travel at different speeds, the distance from any one sensor to an individual source is computed from the difference in its $\mathrm{P}$ and $\mathrm{S}$ wave arrival times. With three or more sensors (or fewer if wave directions are estimated from $P$ and $S$ polarization), the location of the source can be triangulated. The method is labor intensive because of the analyst interaction required to identify and time the wave onsets.

their onsets can be accurately timed. Often transient signals are not detected, or are detected but cannot be timed [Thorne and Morris, 1988a].

Another commercially-available microseismic technique logs the fracture height from the treatment well following the fracturing process. Known as the CMR method [Fix et al., 1989], this method uses the Continuous Microseismic Radiation emitted by the fracture system to delineate the height of the fracture. The approach uses a single clamped threeaxis geophone in the treatment well. The fracture is delineated by moving the geophone in the well, to sample the CMR above, in and below the injection horizon. The physical principle exploited by the CMR method is that seismic waves propagating from the fracture back to the treatment well will be preferentially polarized in the horizontal axes of a threecomponent instrument. The ratio of the horizontal-axis CMR power to the vertical-axis 
CMR power is abnormally large when the tool is adjacent to the fracture. The vertical extent of an anomaly in the ratio indicates the extent of the fracture.

The CMR method is attractive because it does not require manual timing, requires only simple computations, and can be completed shortly after the fracturing process is concluded. It has a deeper formation penetration capability than the other logging methods mentioned previously, giving a more accurate estimate of fracture height [Hunt, 1991]. The principal disadvantage of the method is that it provides only a height estimate.

\subsection{Proposed Hydraulic Fracture Imaging Method}

In principle, the continuous microseismic radiation field should contain significantly more information about the location of sources than is extracted by the CMR ratio method. Continuous noise-like signals are used to construct images of emitter locations in many applications. For example, images of astronomical radio emitters are constructed from noiselike signals using radio telescopes across the globe as elements of very-long-baseline interferometers. Coherent array processing methods are used to locate submarines using narrowband continuous signals recorded on sonar hydrophone arrays [Baggeroer et al., 1988; Knight, Pridham and Kay, 1981].

We propose to adapt coherent array processing methods to map the distribution of CMR emitters, and so to detect the extent of a fracture system (Fig. 3). Our approach uses narrowband signal processing methods, relying upon the relative phase delays induced by wave propagation across an array of hydrophones or clamped geophones to locate emitters. The method is potentially fast because it does not require analyst-intensive timing measurements on the data. As our simulations show, it should operate under conditions of low SNR. It appears likely that the method is viable even under situations where the signal is two to four times smaller than the ambient noise at any one sensor. The method does require an estimate of the velocity structure of the medium, and is sensitive to errors in the assumed velocity structure. However, we demonstrate in our simulations that velocity errors as large as $10 \%$ may not prohibit useful reconstructions of the emitter distribution.

For sake of concreteness, we discuss in detail the monitoring sensor geometry shown in figure 4. The downhole array is proposed for imaging acoustic or elastic emissions from the growing fracture. The two surface clusters of geophones near the treatment wellhead and the measurement wellhead are intended as auxiliary noise references for suppressing noise propagating from the surface and picked up by the downhole sensors. Cancellation has been used with some success by Fix et al. [1989] to cancel tube waves converting from ground roll at the surface and propagating down to a borehole sensor. Cancellation has also been used to suppress noise propagating as body waves ( $\mathrm{P}$ and $\mathrm{S}$ ) from machinery to nearby seismic arrays [Harris, Jarpe and Harben, 1991]. It is likely that the pumping equipment used to perform hydraulic fracturing often is a major noise source that could interfere with signal reception at downhole sensors. Depending on the amount of attenuation present in the medium, noise could propagate from the pumps to the downhole sensors either directly as body waves, or indirectly as surface waves converting to tube waves at the measurement wellhead (then down the measurement well to the array). We will not dis- 


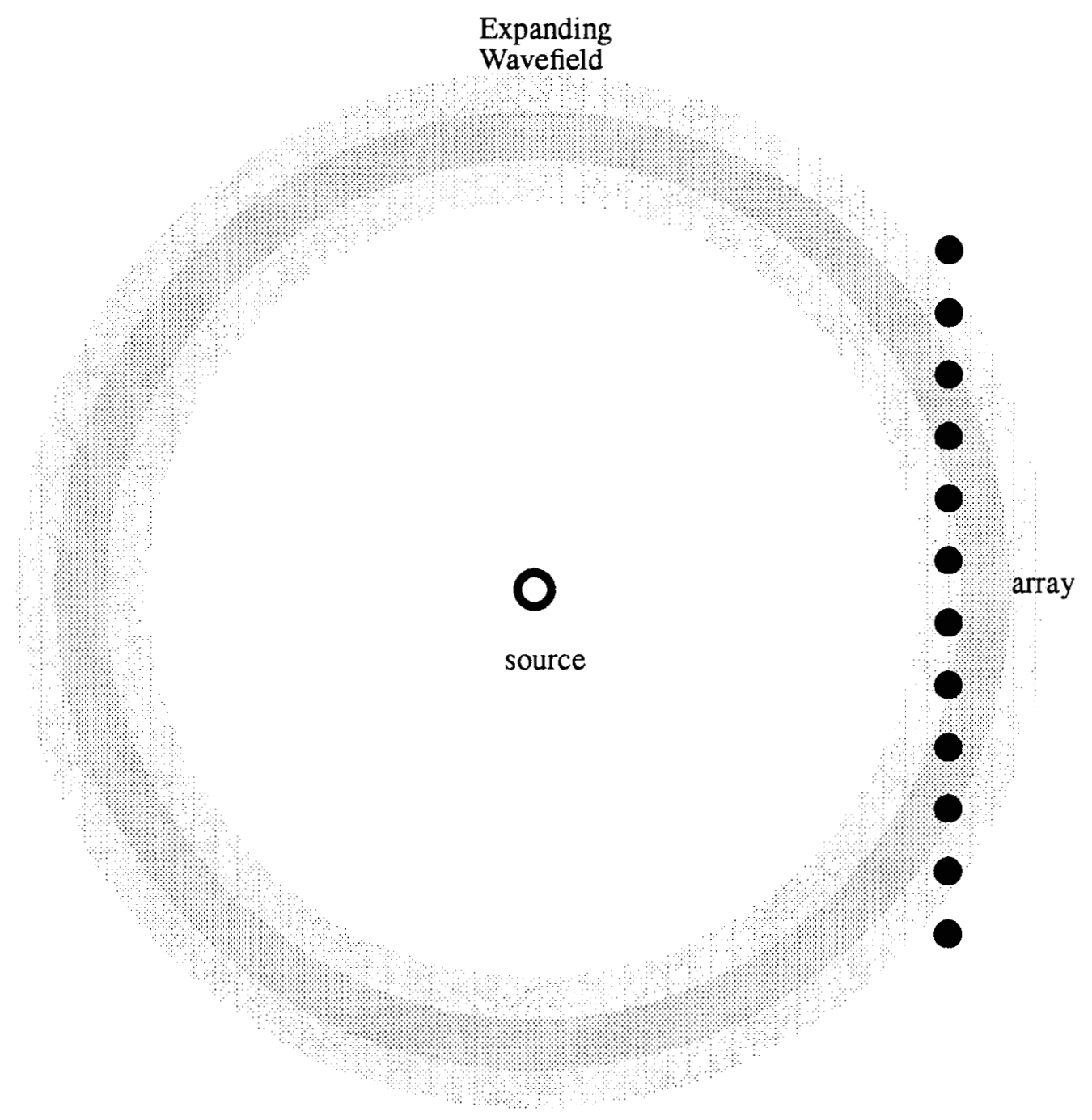

Figure 3 We propose coherent array processing methods to locate microseismic sources. The sources may generate either transient time signals or continuous noise-like signals. Coherent array processing methods (near-field beamforming) estimate the distance to a source by measuring the curvature of the wavefront as it passes the array. The more distant an emission source, the greater the radius of curvature. A vertical array, as depicted here, estimates the vertical position of the source by detecting which phones the wave arrives at first. A vertical array is capable of locating sources in a plane. Several vertical arrays in separate boreholes are capable of locating sources uniquely in a volume. 


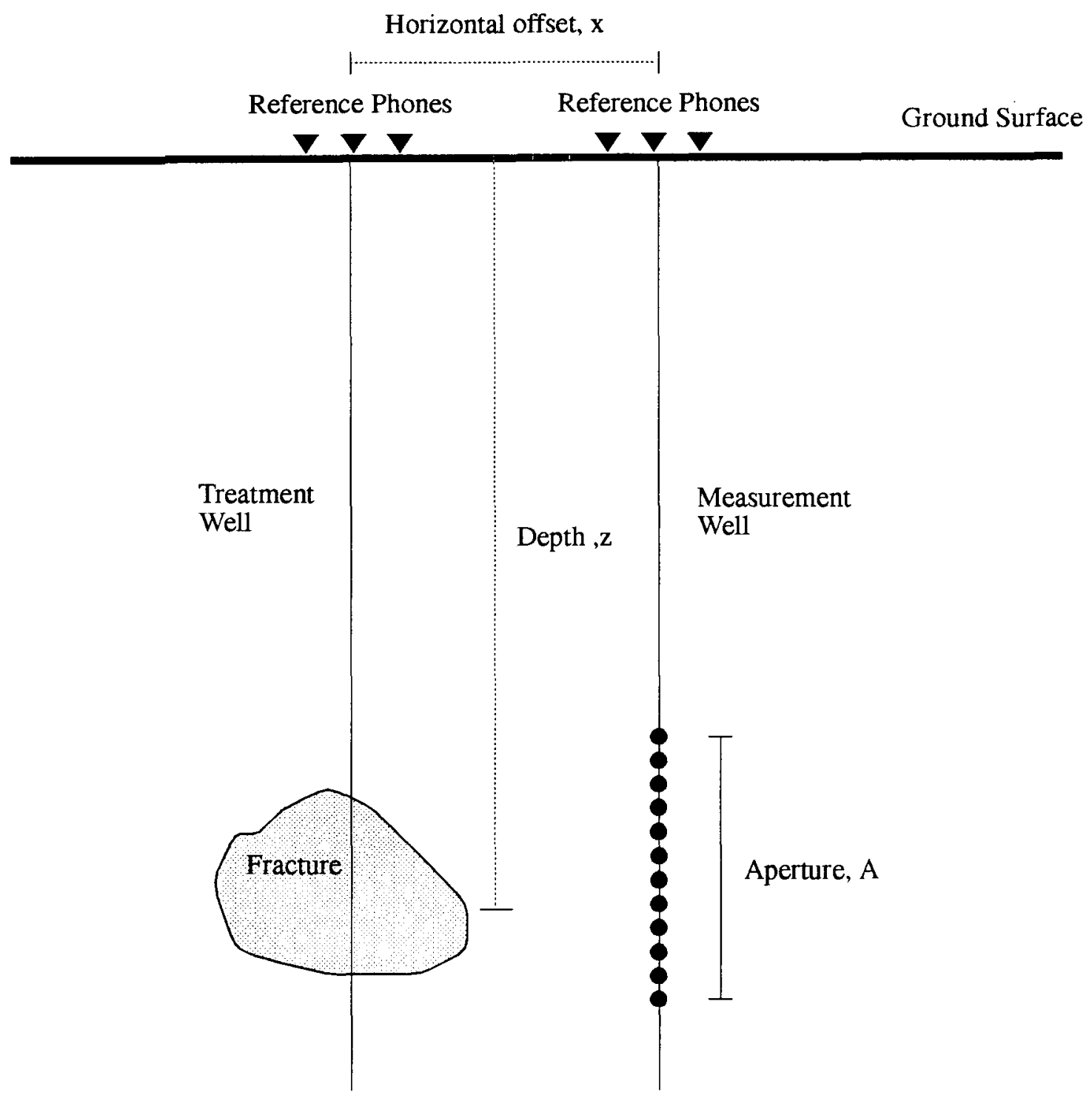

Figure 4 Sensor geometry for monitoring hydraulic fracture development discussed in this report. The concept uses a down-hole array of 12 sensors. Hydrophone arrays of this size are commercially available, although arrays of clamped geophones are preferable. Two small surface arrays of reference geophones can be placed around the pumping equipment near the treatment wellhead and around the measurement wellhead. Reference phones are potentially useful for cancelling pumping noise originating at the surface and propagating either through the formation as body waves, or converting from ground roll at the measurement wellhead and propagating down the measurement well as tube waves.

cuss cancellation further in this report. Instead we concentrate on the imaging aspects of the problem.

At present, hydrophone arrays of the necessary size for the methods we propose are commercially available. These have the advantage that they are easy to deploy and are relatively inexpensive. However, hydrophones have the considerable disadvantage of being indirectly coupled to the medium. Consequently, they are sensitive to tube wave noise. Body waves incident on the borehole convert to tube waves before they are detected by 
hydrophones, which may severely complicate array processing. Arrays of clamped geophones that should mitigate this problem are under development [Marzetta et al., 1988]. In this report we will neglect the effects of tube wave conversions within the array to assess the ultimate potential of monitoring with well-coupled sensors.

\subsection{Overview of Theoretical Discussion and Simulations}

We present the theoretical basis for imaging emitters using CMR in the next section (2). Section 2 can be skipped without loss of continuity, by those not interested in the details of array signal processing. The details include a description of beamforming, upon which all array processing methods are based, as well as a discussion of efficient and high-resolution narrowband methods. We discuss narrowband models for signal and noise that we use to simulate mapping performance. We also discuss a method to correct for unknown wave amplitudes at the downhole sensors. It is often the case that travel times are better known in a heterogeneous medium than are wave amplitudes. We explore the possibility of jointly estimating wave amplitudes and emitter locations.

While simple beamforming techniques can be developed from spherical spreading assumptions appropriate to uniform velocity structures, the problem of locating emitters in heterogeneous media requires a more general approach. Matched field processing [cf. Baggeroer et al., 1988] is the appropriate generalization of beamforming. It requires knowledge of the velocity structure, and the ability to perform forward calculations of the acoustic (or elastic) fields generated by emission sources and measured at the receiver array. As a tutorial, we show how this method generalizes directly from beamforming.

Section 3 contains the results of extensive simulations. We use simulations to study the appropriate frequency band and array location for obtaining usable images of emitter locations. Our objective is to ascertain the best relative depth and distance between the array and the fracture for purposes of imaging the fracture. We also examine the benefits of frequency averaging, a simple form of wideband processing. Frequency averaging helps reject the artifacts associated with narrowband processing and insufficient sensor density (spatial aliasing). Finally, we use simulations to demonstrate matched field processing for the acoustic case. Having a matched field processing capability allows us to examine the effects of model error, i.e. the effects of using an incorrect velocity structure and associated computed field to locate emitters.

In Section 4, we discuss strategies for extending matched field processing to elastic propagation, and tomographic methods for exploiting the calibration potential of perforation shots. 


\subsection{Theory}

\subsection{Assumptions}

For convenience, we introduce coherent array processing methods for acoustic propagation. We expect that wideband elastic waves will be emitted continuously from expanding fractures, and so sketch the elastic extension of the matched field processing approach in our concluding section.

We assume that the phase and amplitude of emission sources are uncorrelated from one frequency to the next. This assumption basically states that the emitter waveforms are random. It is convenient because it allows us to process the wideband data as a set of narrowband signals without any loss to detection or mapping performance. As we shall see, the narrowband assumption is very convenient when dealing with array data, because the temporal and spatial dependence of the array signals are separable. The random signal assumption allows us to process the data as multiple simple narrowband components, then average the resulting maps of emitter power over frequency for a wideband result. A complex analytic narrowband signal and noise representation [see Knight et al., 1981] further simplifies mathematical manipulations.

\subsection{Beamforming}

In a uniform acoustic medium, waves emitted from a point source propagate spherically outward. The signal recorded by a sensor located at position $\chi$ in the presence of a source at location $\xi$ with excitation time history $f(t)$, and in the presence of ambient background noise is:

$$
s(t)=\frac{1}{r} f\left(t-\frac{r}{v}\right)+n(t)
$$

Here $r$ is the distance between the source and receiver:

$$
r=\|\underline{\xi}-\underline{\chi}\|
$$

and $v$ is the medium velocity. An array of $N$ sensors at locations $\underline{\chi}_{i}$ will produce the recorded signals:

$$
s_{i}(t)=\frac{1}{r_{i}} f\left(t-\frac{r_{i}}{v}\right)+n_{i}(t)
$$

Under certain statistical assumptions for the noise ${ }^{(1)}$, the beamforming operation:

$$
b(t)=A \cdot \sum_{i=1}^{N} \frac{1}{r_{i}} s_{i}\left(t+\frac{r_{i}}{v}\right) \quad A=\left[\sum_{i=1}^{N}\left(\frac{1}{r_{i}}\right)^{2}\right]^{-1}
$$

(1) independent, zero mean, white, identically distributed Gaussian random processes 
provides the maximum likelihood estimate (called the beam) for the signal emitted by the source given the array data. Observe that the beamforming operation entails weighting, shifting and summing the array signals. The signals are shifted to eliminate the delays due to propagation across the array. They are weighted to match the amplitude pattern across the sensors caused by geometric spreading. When summed, the signal component of the received waveforms add coherently, and the ambient noise components interfere destructively. The factor $A$ is a normalization to ensure that the beam equals the source excitation when no noise is present.

Beamforming requires knowledge of the source location to calculate the appropriate weights and steering delays. When the location of the source is unknown, it is common to scan the beam over a "search" region of candidate source locations. Commonly, the energy in the beam:

$$
E(\underline{\xi})=\left\{b^{2}(t) d t\right.
$$

is maximized over a time interval $I$ of duration $T$ seconds to locate the source. If multiple sources are present, this energy functional is typically large wherever a source exists. Consequently, a distributed source of acoustic emissions can be imaged by beamforming operations on the data.

\subsection{Narrowband Propagation Models}

The shifting operation is time consuming when performed on large data sets. A substantial reduction in the computational cost of repeated beamforming operations is possible if beamforming is conducted on narrowband signals. Filtering the data into narrow frequency bands has the effect of decoupling the spatial and temporal variations of propagating wavefields. This is highly desirable for mapping fracture development, since we are interested only in the spatial distribution of emitters, not the temporal characteristics of the signals they generate. The narrowband transformation converts the shifting operation to multiplication by a complex phase delay factor. This feature of the transformation accounts for major increases in computational speed.

\subsubsection{Single Source, Spherical Propagation}

Neglecting noise, if the signal of equation 3 is filtered into a very narrow frequency band, with center frequency $\omega$, it assumes the form of a modulated sinusoid:

$$
s_{i}(t) \approx \frac{1}{r_{i}} a\left(t-\frac{r_{i}}{v}\right) \cos \left[\omega\left(t-\frac{r_{i}}{v}\right)+\phi\left(t-\frac{r_{i}}{v}\right)\right]
$$

The amplitude $a(t)$ and phase $\phi(t)$ of the source are slowly varying functions. They do not change significantly on time scales much less than the reciprocal of the filtering bandwidth. If relative propagation times across the array are this small, i.e. if the array aperture 
and processing bandwidth are small enough, then the signal amplitude and phase do not change significantly across the array aperture:

$$
a\left(t-\frac{r_{i}}{v}\right) \approx a(t) \quad \phi\left(t-\frac{r_{i}}{v}\right) \approx \phi(t)
$$

The result is that the signals received across the array take the simpler form:

$$
s_{i}(t) \approx \frac{1}{r_{i}} a(t) \cos \left[\omega\left(t-\frac{r_{i}}{v}\right)+\phi(t)\right]
$$

This signal may be represented as the real part of a complex signal, called the complex analytic representation:

$$
s_{i}(t) \approx \operatorname{Re}\left\{z_{i}(t) e^{j \omega t}\right\}=\operatorname{Re}\left\{\frac{1}{r_{i}}\left[a(t) e^{j \phi(t)}\right] e^{-j \omega \frac{r_{i}}{v}} e^{j \omega t}\right\}
$$

The complex analytic representation can be constructed from the narrowband signal in two steps: first the narrowband signal is phase-shifted $90^{\circ}$, then the narrowband signal and its phase-shifted (quadrature) counterpart are combined as the real and imaginary parts, respectively, of the complex signal. A spectral interpretation of the transformation is shown in figure 5 .

When grouped as a vector, the collection of array signals takes on a yet simpler form:

$$
\underline{s}(t) \approx \operatorname{Re}\left\{\underline{z}(t) e^{j \omega t}\right\} \quad \underline{z}(t)=\left[a(t) e^{j \phi(t)}\right] \underline{\varepsilon}(\underline{\xi})
$$

where

$$
\underline{\varepsilon}\left(\underline{\xi)}=\left[\begin{array}{c}
\frac{1}{r_{1}} e^{-j \omega \frac{r_{1}}{v}} \\
\bullet \\
\bullet \\
\frac{1}{r_{N}} e^{-j \omega \frac{r_{N}}{v}}
\end{array}\right]\right.
$$




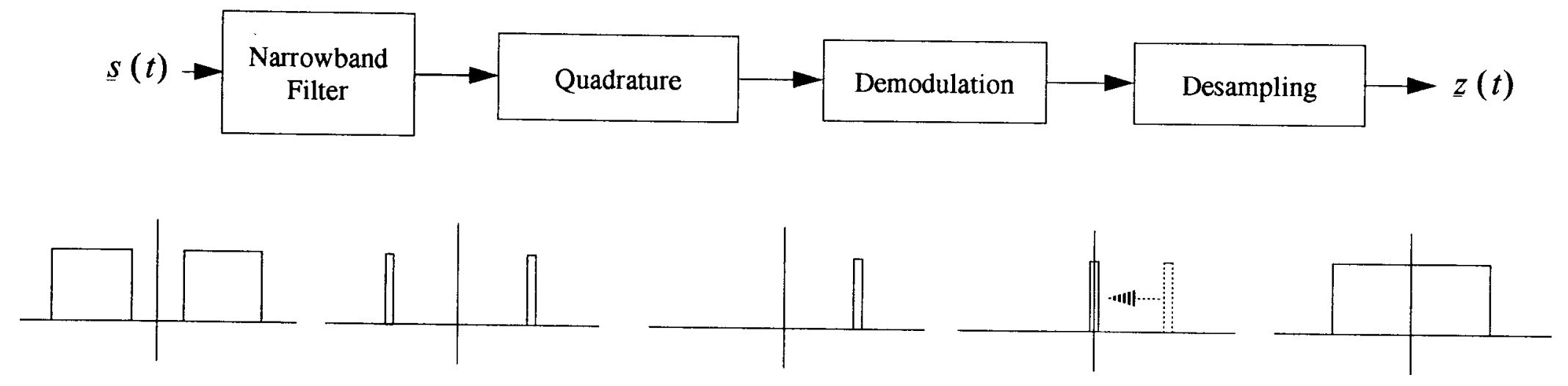

Signal Spectrum

Data Covariance Calculation:

$$
\underline{R}=\int_{t} z(t) z^{H}(t) d t
$$

Figure 5 Spectral interpretation of the complex analytic transformation performed on the data to speed beamforming operations and facilitate high-resolution methods. First, the data are filtered into a narrow frequency band. The negative frequencies are eliminated to create a complex (analytic) signal. This signal is demodulated to baseband (zero frequency), then down-sampled to eliminate oversampling. 
The vector $\varepsilon(\xi)$ is called the steering vector, since it incorporates the collection of complex phase delays and amplitudes induced by propagation across the array. It incorporates the spatial structure of the array signal. Note that equation 10 has effected a decomposition of the signal into a product of separable temporal and spatial factors.

The beamforming operation can be expressed as a simple algebraic operation using the signal model of equation 10 . Under the narrowband assumption,

$$
b(t)=\operatorname{Re}\left\{\underline{w}^{H} \underline{z}(t) e^{j \omega t}\right\} \quad \underline{w}=\frac{\underline{\varepsilon}(\underline{\xi})}{(\underline{\varepsilon}(\underline{\xi}))^{H} \underline{\varepsilon}(\underline{\xi})}
$$

The superscript $H$ denotes the Hermitian (conjugate) transpose operation. The complicated shift-and-sum operation of beamforming is reduced to a simple dot product with the steering vector. Finally, the energy in the beam can be written in the simple form:

$$
\begin{gathered}
E\left(\underline{\xi)}=\frac{1}{2} \int_{\underline{\underline{w}}}\left|\underline{w}^{H} \underline{z}(t)\right|^{2} d t=\frac{1}{2} \underline{w}^{H} \underline{R} \underline{w}\right. \\
\underline{R}=\int_{Y} \underline{z}(t)[z(t)]^{H} d t
\end{gathered}
$$

The matrix $R$ is called the sample spatial covariance matrix of the array signal. Note that the energy in the beam is calculated from $z(t)$, which depends only on the slowly varying phase and amplitude envelopes of the source signals. It is possible to demodulate and desample the complex analytic representation of the received signal, to significantly increase the computational speed of the beamforming operation (see figure 5 and [Knight, Pridham and Kay, 1981]).

\subsubsection{Distributed Source, General Medium - Matched Field Processing}

In practice, sites of acoustic or elastic emissions will be distributed over large portions of the fracture volume. We need a more general model for propagation to the array through complicated media. Consequently, the complex narrowband signal received across the array is more correctly an integral over the volume $\Omega$ of emission locations:

$$
\underline{z}(t)=\int_{\Omega} f(t, \underline{\xi}) \underline{\varepsilon}(\underline{\xi}) d \underline{\xi}+\underline{n}(t)
$$

where $f(t, \xi)$ is a source driving function at location $\xi$ and the $\varepsilon(\xi)$ is the vector of monochromatic Green's functions characterizing propagation from the source to each of the $N$ array sensors: 


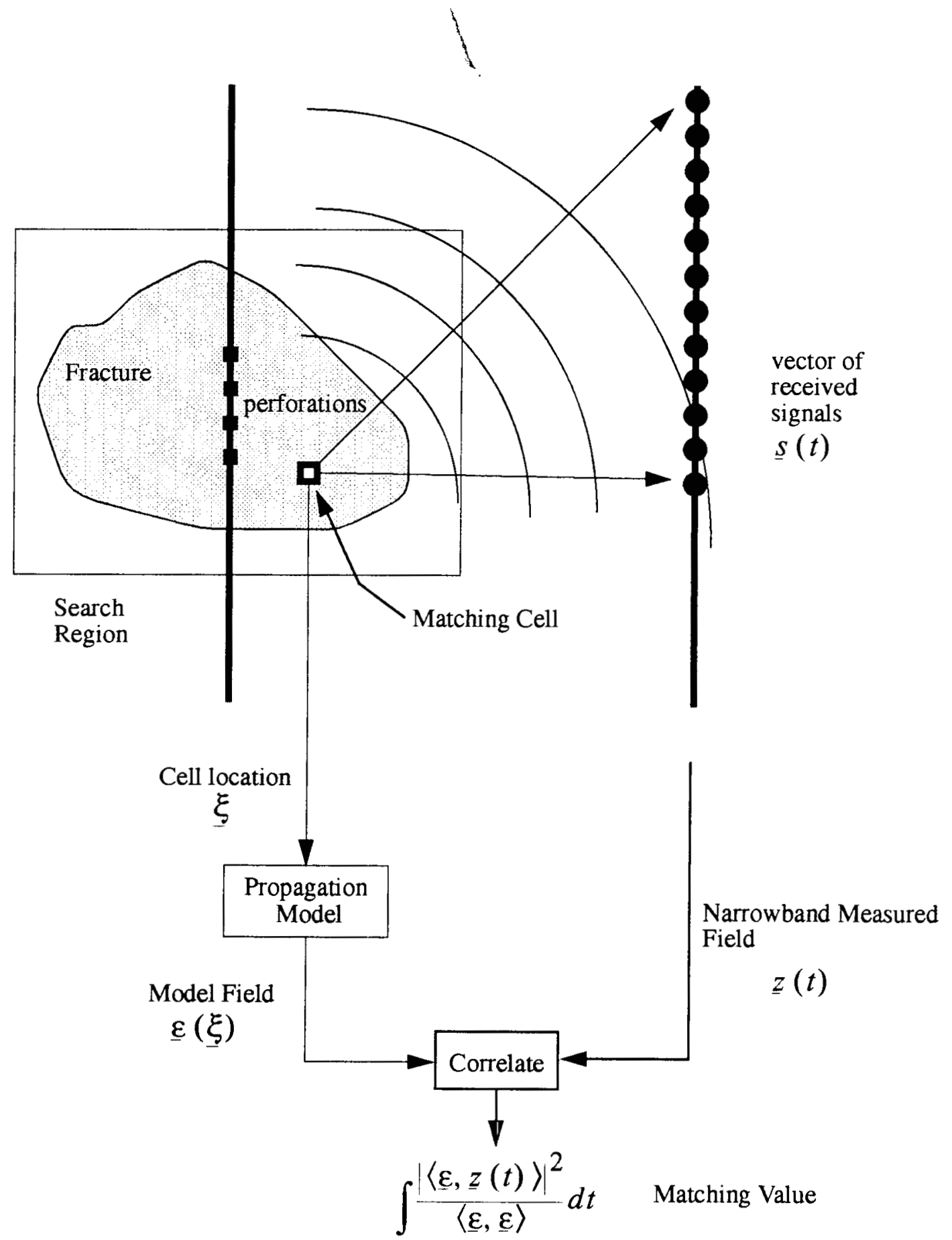

Figure 6 Matched field processing compares a measured field to a synthetic field generated by a propagation model. It computes a matching value as a function of an assumed source location. The matching value is displayed as an image over a search region, and, in principal, is large wherever a source exists. 


$$
\underline{\varepsilon}(\underline{\xi})=\left[\begin{array}{c}
\alpha_{1} e^{i \Psi_{1}(\xi)} \\
\bullet \\
\bullet \\
\alpha_{N} e^{i \Psi_{N}(\xi)}
\end{array}\right]
$$

The phase terms $\Psi_{i}(\xi)$ result from propagation phase delays, and the $\alpha_{i}(\xi)$ represent corresponding wave spreading amplitudes. For general media, the narrowband Green's functions are not representable in closed form, as they were in the last section for simple spherical waves.

Matched field processing [cf. Baggeroer et al., 1988] is the approach that results from substituting the vector of Green's functions (equation 16) for the simple steering vector of equation 11. It generalizes the beamforming operation of equation 13 to work in complicated media. The method is summarized in figure 6.

Generally, analytical expressions are not available for the Green's functions. They must be evaluated by solving the wave equation with a numerical technique such as a finite difference method. We use a split-step method to solve a paraxial approximation of the wave equation [Thomson and Chapman, 1983]. The paraxial method models propagation in one direction only (from the source to the receiver). Very efficient spectral implementations for paraxial wavefield extrapolation exist. The FFT split-step method we use is one such method. Since we solve the narrowband problem, we obtain a further reduction in computational complexity by eliminating the time dimension from the solution.

\subsubsection{Models for Simulation}

For purposes of simulation, we treat the signals and noise as continuous zero-mean random processes assuming Gaussian statistics. We also assume that sources at different locations emit statistically independent signals, i.e.:

$$
E\left\{f\left(t, \underline{\xi}_{1}\right) f\left(t, \underline{\xi}_{2}\right)\right\}=\sigma_{S}^{2}\left(\underline{\xi}_{1}\right) \delta\left(\underline{\xi}_{1}-\underline{\xi}_{2}\right)
$$

where the symbol $E$ denotes the statistical expectation operator, and $\sigma_{S}^{2}(\xi)$ is the power emitted at location $\xi$. A series of discrete sources will generate the received field:

$$
\underline{z}(t)=\sum_{i} f\left(t, \underline{\xi}_{i}\right) \underline{\varepsilon}\left(\underline{\xi}_{i}\right)+\underline{n}(t)
$$

The noise $n(t)$ is assumed to be independent of the signal and of two types: sensor "self noise" which is uncorrelated from sensor to sensor, and tube wave noise which propagates up and down the measurement well. Tube wave noise is correlated from sensor to sensor. The simplest possible tube wave noise would originate at points above and below the ar- 
ray and propagate past it at the tube wave velocity $v_{t}$ (about $5000 \mathrm{ft} / \mathrm{sec}$ ). A suitable model for this simplest case is:

$$
\underline{n}(t)=\underline{Q}\left[\begin{array}{l}
q_{1}(t) \\
q_{2}(t)
\end{array}\right]
$$

where $q_{1}$ and $q_{2}$ are excitation envelopes of the down- and up-going waves respectively, and the $N \times 2$ matrix

$$
Q=\left[\begin{array}{cc}
1 & \zeta^{(N-1)} \\
\zeta & \zeta^{(N-2)} \\
\bullet & \bullet \\
\bullet & \bullet \\
\zeta^{(N-1)} & 1
\end{array}\right]
$$

with

$$
\zeta=\beta e^{-j \frac{\omega}{v_{t}} \Delta z}
$$

contains the vectors of propagation delays for the tube waves, for the $N$ equally spaced sensors (spacing $\Delta z \mathrm{ft}$ ). The complex exponential phase factor represents the time delay $(\Delta z) / v_{t}$ from one sensor to the next and the real factor $\beta$ is an attenuation factor $(0<\beta<1)$.

To simulate the sample covariance matrix $R$ used in beamforming, we generate samples of the array signals $z(t)$ at intervals $m \Delta t$ We use a Gaussian random number generator to provide the necessary samples $f_{i}(m \Delta t)$ and $q_{i}(m \Delta t)$, as well as samples of independent noise on each of the sensors. The sampling interval for uncorrelated sequential samples is $\Delta t=1 / B$ where $B$ is the width in Hertz of the narrow filter band used for analysis. The total number of samples is $M \approx T B$ to represent an integration time of $T$ seconds. The integration time is limited to be less than the expected temporal scale of changes in the distribution of emitters. Over such limited time periods, the spatial structure of the signal should be unchanging, and it is appropriate to average signal statistics. For the hydraulic fracturing application, the integration time should be smaller than the amount of time it takes the fracture to traverse a beamforming resolution cell. This time duration may be on the order of a few tens of seconds.

The sample covariance matrix will tend to a limiting value as the integration time is increased: 


$$
\lim _{T \rightarrow \infty} \frac{1}{T} \underline{R}=\underline{K}=E\left\{\underline{z}(t) \underline{z}^{H}(t)\right\}
$$

We expect that the signals and noise will be statistically independent, so that the covariance of their sum will be the sum of their individual covariances:

$$
\underline{K}=\underline{K}_{S}+\underline{K}_{N}
$$

The signal covariance will be:

$$
\underline{K}_{S}=\int d \underline{\xi}_{1} \int d \underline{\xi}_{2} E\left\{f\left(t, \underline{\xi}_{1}\right) f\left(t, \underline{\xi}_{2}\right)\right\} \underline{\varepsilon}\left(\underline{\xi}_{1}\right) \underline{\varepsilon}^{H}\left(\underline{\xi}_{2}\right)
$$

Under the assumption of emitter independence (equation 17), the signal covariance simplifies:

$$
\underline{K}_{S}=\int_{\Omega} \sigma_{S}^{2}(\underline{\xi})\left(\underline{\varepsilon}(\underline{\xi}) \underline{\varepsilon}^{H}(\underline{\xi})\right) d \underline{\xi}
$$

The noise covariance consists of a simple tube wave term and a spatially uncorrelated sensor self-noise term:

$$
\underline{K}_{N}=\underline{Q} \underline{\underline{Q}} \underline{Q}^{H}+\sigma_{N}^{2} \underline{I}
$$

where $I$ is the identity matrix. The sensor self-noise contributes the second diagonal term, which is the product of the sensor noise power $\sigma_{N}^{2}$ and the identity matrix. The the on-diagonal terms of the matrix $\underline{\Sigma}$ represent the power of the down- and up-propagating tube waves:

$$
\underline{\Sigma}=\left[\begin{array}{cc}
\sigma_{d}^{2} & c_{d u} \\
c_{u d} & \sigma_{u}^{2}
\end{array}\right]
$$

The off-diagonal terms represent any coupling between up- and down-going waves. This model should suffice for tube waves generated at locations outside of the array aperture. The coupling terms might represent reflection off of the top or bottom of the well, and would incorporate the phase delays corresponding to two-way travel time to reflectors.

\subsection{High-Resolution Methods}

A large collection of methods are available for increasing the resolution of narrowband beamforming operations. We use one in our simulations, called the Minimum Variance Distortionless Receiver (MVDR), also known as Capon's maximum likelihood method [Baggeroer et al, 1988]. The MVDR utilizes the fact that narrowband beamforming is a dot product operation (equation 12) between a vector of weights $\underline{w}$ and the received sig- 
nal. The weights are the normalized Green's functions for matched field processing. In the MVDR method, the weights are chosen adaptively to suppress spurious energy generated by strong emitters outside the matching cell from "leaking" into the beam. The beam energy or matched field output

$$
\underline{w}^{H} \underline{R} \underline{w}
$$

is minimized in the MVDR approach subject to a constraint that any signal emitted from the matching cell passes without distortion:

$$
\underline{w}^{H} \underline{\varepsilon}(\underline{\xi})=1
$$

Note that for signals propagating from the "target cell", $\underline{z}(t)=f(t) \underline{\varepsilon}$ and

$$
\underline{w}^{H} \underline{z}(t)=f(t) \underline{w}^{H} \underline{\varepsilon}=f(t)
$$

The weights are readily found to be

$$
\underline{w}=\frac{\underline{R}^{-1} \underline{\varepsilon}}{\underline{\varepsilon}^{H} \underline{R}^{-1} \underline{\varepsilon}}
$$

and the matching value is

$$
\underline{w}^{H} \underline{R} \underline{w}=\frac{1}{\underline{\varepsilon}^{H} \underline{R}^{-1} \underline{\varepsilon}}
$$

Note that the MVDR is very like the original matched field processor, involving computation of a quadratic form. The quadratic form is in the denominator, in this case, and operates on the inverse of the covariance matrix.

\subsection{Compensating for Unknown Amplitudes}

Often it is the case that wave amplitudes are harder to model accurately than travel times. Consequently, we examine a technique for matching wavefields without knowledge of the wave amplitudes at the array sensors. We assume that the phase alone is known from the solution of the wave equation. The steering vector $\varepsilon$ must be corrected with unknown (real) amplitudes to be estimated from the data. This may be accomplished by expressing the steering vector in the form:

$$
\underline{w}=\underline{\Gamma}=\left[\begin{array}{ccc}
\varepsilon_{1} & & 0 \\
\bullet & \\
& \ddots & \\
0 & & \varepsilon_{N}
\end{array}\right]\left[\begin{array}{l}
\gamma_{1} \\
\vdots \\
\vdots \\
\gamma_{N}
\end{array}\right]
$$


where the matrix $\underline{\Gamma}$ is a diagonal matrix with diagonal elements given by elements of the steering vector, and the vector $\gamma$ is a vector of real amplitude compensation coefficients. We choose the unknown coefficients to maximize the matched field energy of equation 13 subject to the constraint $\gamma^{T} \gamma=1$ that prevents the coefficients from becoming arbitrarily large. Substituting equation 33 into equation 13 results in the matched field energy:

$$
E=\underline{\gamma}^{T} \underline{\Gamma}^{H} \underline{R} \underline{\Gamma}
$$

It is straightforward to show that the maximizing amplitude correction vector is the eigenvector of the matrix $R e\left\{\underline{\Gamma}^{H} \underline{R} \underline{\Gamma}\right\}$ that corresponds to the largest eigenvalue. For this choice of amplitude correction vector, the matched field energy is:

$$
E=\lambda_{\max }\left(\operatorname{Re}\left\{\Gamma^{H} R \Gamma\right\}\right)
$$

In a similar fashion, the MVDR method can be modified to compensate for unknown amplitudes. From equation 32, the MVDR estimate of the matched field energy adjusted for the unknown amplitude model is:

$$
E=\frac{1}{\gamma^{T} \underline{\Gamma}^{H} \underline{R}^{-1} \underline{\Gamma} \underline{\gamma}}
$$

The maximum of this expression is obtained by choosing the amplitude correction vector to be the eigenvector of $\operatorname{Re}\left\{\underline{\Gamma}^{H} \underline{R}^{-1} \underline{\Gamma}\right\}$ corresponding to the minimum eigenvalue. In this case, the best estimate of the matched field energy is:

$$
E=\lambda_{\min }^{-1}\left(\operatorname{Re}\left\{\underline{\Gamma}^{H} \underline{R}^{-1} \Gamma\right\}\right)
$$

Because this model has so many more free parameters to fit the data, it may be expected to produce more artifacts in matched field images of emitter distributions.

\subsection{Simulations}

In order to test the imaging performance of beamforming and matched field processing we completed a series of simulations. Our objectives were to determine whether these methods have sufficient resolution to be useful to hydraulic fracture monitoring, what the appropriate experimental geometry is for a field test, and what the effects of propagation model error might be.

\subsection{Choice of array location}

The first issue that we explore with simulations is the appropriate array range and vertical offset for resolving emitters in the near-field. We examine the best placement for an array of 12 sensors with 50 foot spacing, as this is an industry standard. Refering to figure 4 , the horizontal offset (range) and vertical offset (depth) of the array are the parameters to be optimized for best resolution in the target imaging region. In figure 8 we display a matrix 

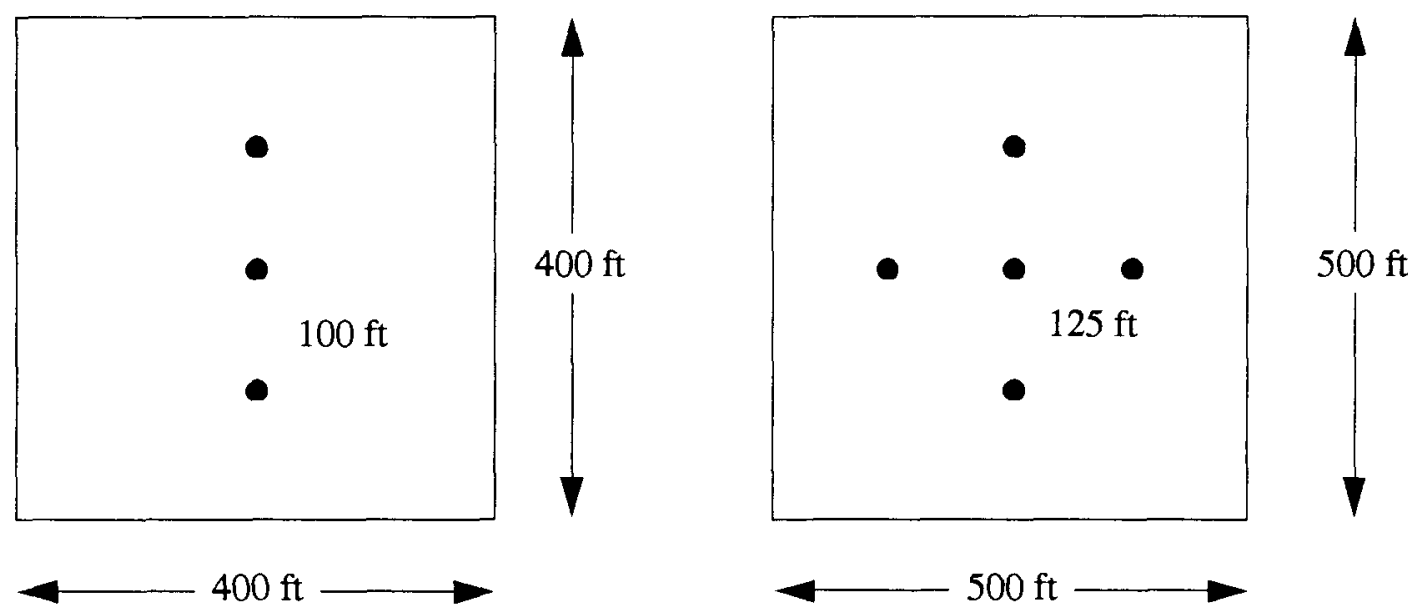

Figure 7 Emitter source patterns for simulations. The pattern to the left is used in the reconstructions of figures 8 and 9 . The pattern to the right is used in the simulated reconstructions shown in figures 10 through 15 . In the latter case, the array center is separated horizontally 400 feet from the center of the emitter distribution.

of matched field reconstructions for three emission sources distributed vertically at 100 foot intervals as shown in figure 7. Reconstructions in the top row are for an array that is centered vertically: the array center coincides with the center emitter depth. The subsequent two rows are for arrays offset by half the array aperture and by more than two apertures in the vertical direction. The propagation speed is assumed to be $2200 \mathrm{ft} / \mathrm{sec}$. in this figure, which is a shear-type velocity in diatomite. The reconstruction frequency is 100 $\mathrm{Hz}$, and the reconstruction method is simple beamforming (equation 13). The best resolution is obtained when the array is centered and situated at a range between 200 and 400 feet. Resolution is poor for substantial vertical offsets, and at close and distant ranges.

The second simulation series (figure 9) is identical in all respects except that the medium and reconstruction velocities have been increased to $5000 \mathrm{ft} / \mathrm{sec}$. to emulate compressional propagation in a diatomite. Best results are still obtained for a vertically centered array about 200 to 400 feet from the sources. Resolution is poorer in this case, because the increased velocity results in an increased wavelength.

\subsection{Benefits of frequency averaging}

Single-frequency reconstructions contain many artifacts (sidelobes) that may be caused by an array that undersamples spatially. Array elements should be spaced at intervals of one-half the shortest wavelength present in the data. An array with 50 foot spacing is undersampled generally at such low velocities $(2000-5000 \mathrm{ft} / \mathrm{sec})$ and at frequencies above $100 \mathrm{~Hz}$. One approach to suppressing artifacts is based upon the observation that the positions of artifacts are frequency dependent. If matched field images are averaged over frequency, the artifacts may fail to superimpose. 


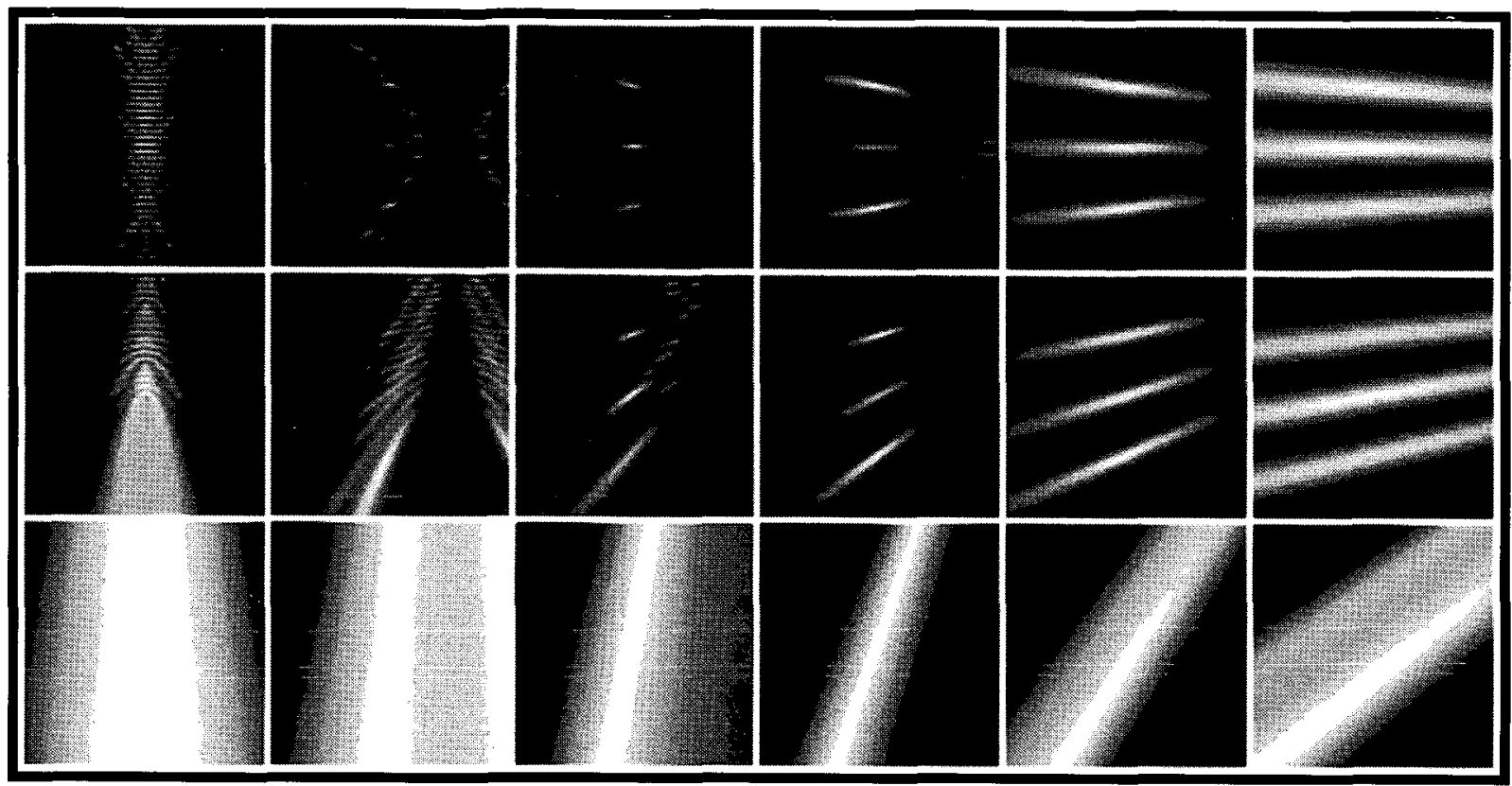

$0 \mathrm{ft}$. $100 \mathrm{ft}$.

$200 \mathrm{ft}$.

$400 \mathrm{ft}$.
Reconstruction Parameters

Sources: 3 on vertical axis Receivers: Linear array of 12 Receiver spacing: $50 \mathrm{ft}$.

Receiver range: 0 to $1600 \mathrm{ft}$. from T. W

Grid size: $400 \mathrm{ft}$. $400 \mathrm{ft}$

Grid spacing: $4 \mathrm{ft}$. $\times 4 \mathrm{ft}$.

\section{Reconstruction method: Beamforming}

Propagation model: Uniform

Geometric attenuation: None

Intrinsic attenuation: None

Amplitude compensation: None

Display: Magnitude
Receivers

Centered

Receivers

$275 \mathrm{ft}$. high

(= 0.5 length of receiver array)

Receivers

$1225 \mathrm{ft}$. high

(Top of array at $3000 \mathrm{ft}$., Center of frac at $4500 \mathrm{ft}$.)

Distance between Treatment Well and Receiver Well

Figure 8 Receiver position has a major effect on S-wave reconstructions. The matrix of reconstructions results from a range of horizontal ( $(0$ to $1600 \mathrm{ft}$.) and vertical ( 0 to $1225 \mathrm{ft}$.) array-source offsets. Best results occur with no vertical offset and a $200 \mathrm{fo} 400 \mathrm{ft}$. horizontal offset. 


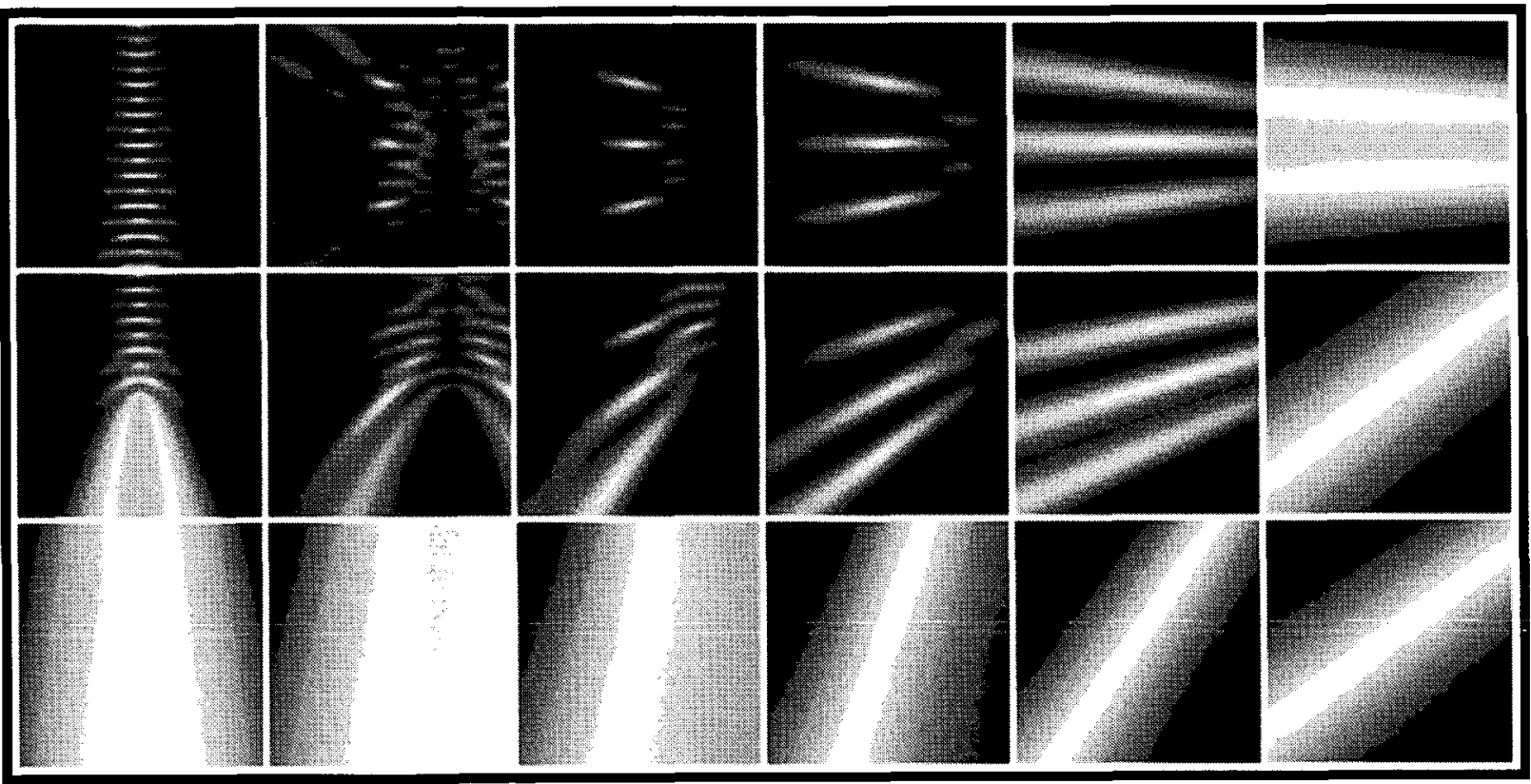

Receivers

Centered

Receivers

$275 \mathrm{ft}$. high

(=0.5 length of receiver array)

Receivers

$1225 \mathrm{ft}$. high

(Top of array at $3000 \mathrm{ft}$.

Center of frac at $4500 \mathrm{ft}$.)
$0 \mathrm{ft}$.
$100 \mathrm{ft}$.
$200 \mathrm{ft}$.
$400 \mathrm{ft}$.
$800 \mathrm{ft}$.
$1600 \mathrm{ft}$.
Geometry
Sources: 3 on vertical axis Receivers: Linear array of 12
Receiver spacing: $50 \mathrm{ft}$. Receiver range: 0 to $1600 \mathrm{ft}$. from $\mathrm{T}$. W. Grid size: $400 \mathrm{ft}$. $x 400 \mathrm{ft}$.
Grid spacing: $4 \mathrm{ft}$. $\times 4 \mathrm{ft}$.
Reconstruction Parameters
Reconstruction method: Beamforming
Propagation model: Uniform
Geometric attenuation: None
Intrinsic attenuation: None
Amplitude compensation: None
Display: Magnitude
Signal Parameters
Tube noise: None
Uncorrelated sensor noise: None
Source amplitude variation: None
Velocity: $5000 \mathrm{ft} . / \mathrm{sec}$. (P-wave)
Frequency: $100 \mathrm{~Hz}$
Time-bandwidth product: Infinity

Distance between Treatment Well and Receiver Well

Figure 9 Optimum receiver position is unchanged when medium velocities increase (c.f. figure 8). P-wave reconstructions are best for no vertical array offset and a 200 to $400 \mathrm{ft}$. horizontal separation. Higher velocities degrade resolution. 
Figure 10 shows the result of altering the reconstruction frequency, and of frequency averaging. The left six columns in this matrix of beamforming images show single-frequency reconstructions at $75,100,125,150,175$, and $200 \mathrm{~Hz}$. The right two columns show the images that result from averaging over these six frequencies and over twenty-six frequencies ( $5 \mathrm{~Hz}$ spacing) in the $75-200 \mathrm{~Hz}$ band. Each row of images shows a different signalto-noise ratio, ranging from almost no noise on the top to an SNR of 0.1 on the bottom. The noise was a combination of a small amount of uncorrelated noise, and a much larger amount of tube wave noise. Consequently, the tube wave noise controlled the SNR in each reconstruction. The conventional beamforming method was used with a centered array 400 feet from the center of the sources. Five sources were present in a cross pattern.

The images produced by frequency averaging clearly show the five sources at a signal to noise ratio of 0.5 . This point is interesting because transient methods would be inoperable when the signal is smaller than the noise on any one sensor. Increased frequency averaging (26 frequencies) at smaller frequency intervals considerably improves the reconstruction, providing a usable image at an SNR of 0.1 . Artifacts overwhelm the six-frequency average below an SNR of 0.25 . Note, in addition, that the nearer emitters are better resolved than the more distant sources.

\subsection{Effects of reconstruction velocity error}

The ability to map emitters with beamforming and matched field processing depends on the availability of a good velocity model. Our next simulations examine the effects of velocity model error.

\subsubsection{Uniform media}

Perhaps the simplest error occurs for a constant-velocity medium when the reconstruction velocity is also constant, but in error. Figure 11 displays the effects of reconstructing our 5 -point pattern of emitters with medium velocities that are $10 \%$ lower than the reconstruction velocity, equal to the reconstruction velocity and $10 \%$ higher. The reconstructions are six-frequency averages in the manner of figure 10 . The correct velocity is $2200 \mathrm{ft} / \mathrm{sec}$., characteristic of shear wave propagation in diatomite. The noise consists of tube waves at an SNR of 0.5. Both the beamforming reconstructions and the MVDR reconstructions are displayed in figure 11. In both cases, the basic cross pattern of sources is preserved when the velocity is in error, but the pattern shifts. When the reconstruction velocity is too high, the pattern moves toward the array. When it is too low, the pattern moves away from the array.

The sense of the movement is counterintuitive, but is explained in the following way. Coherent array processing estimates range by measuring the curvature of the wavefront incident on the array. The curvature is sensed from the pattern of relative time delays across the array. Large relative time delays can be produced by either (1) a relatively distant source emitting waves which propagate at low velocity, or (2) a relatively near source emitting waves which propagate at a higher velocity. If the reconstruction velocity is cho- 


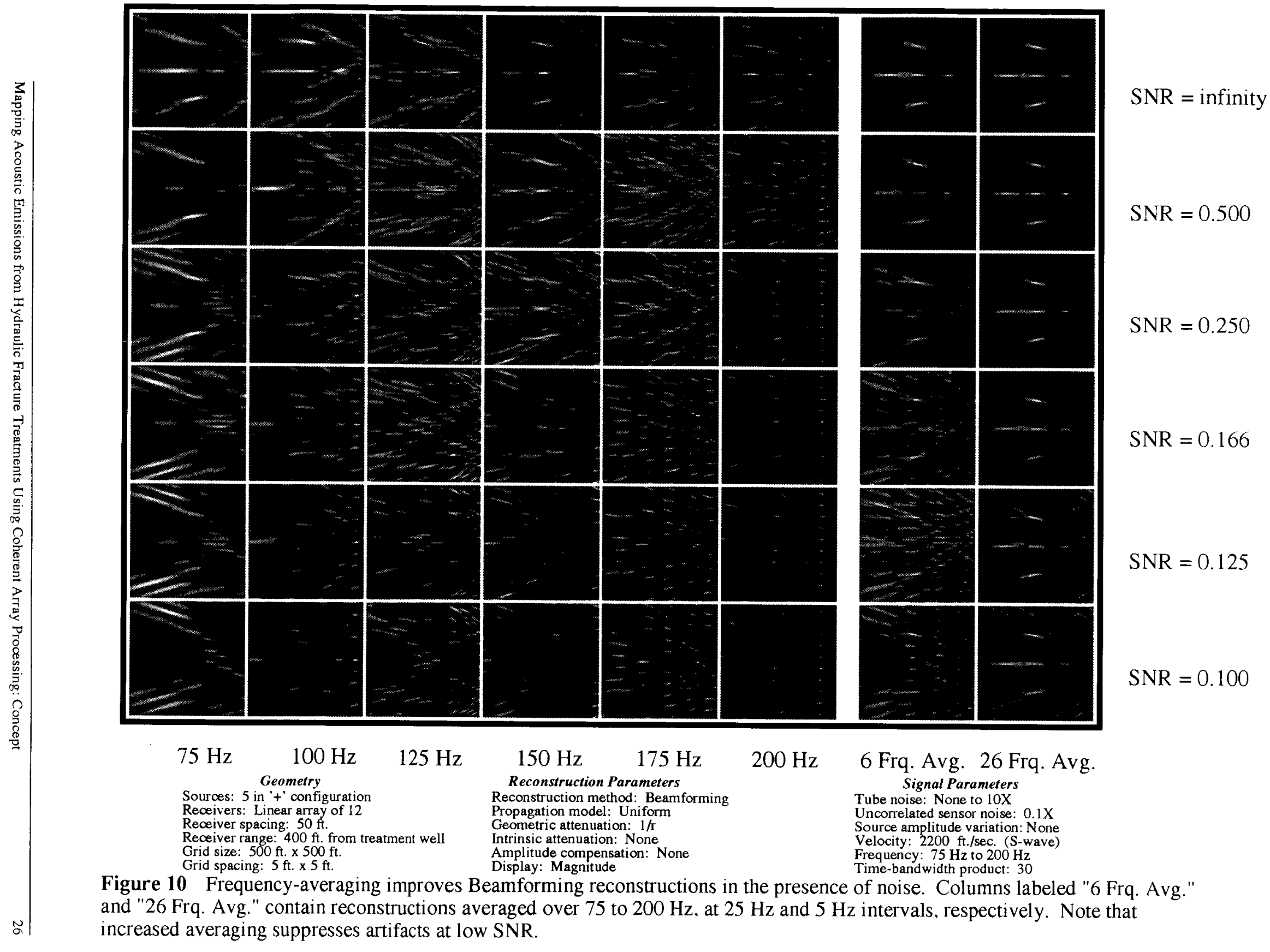



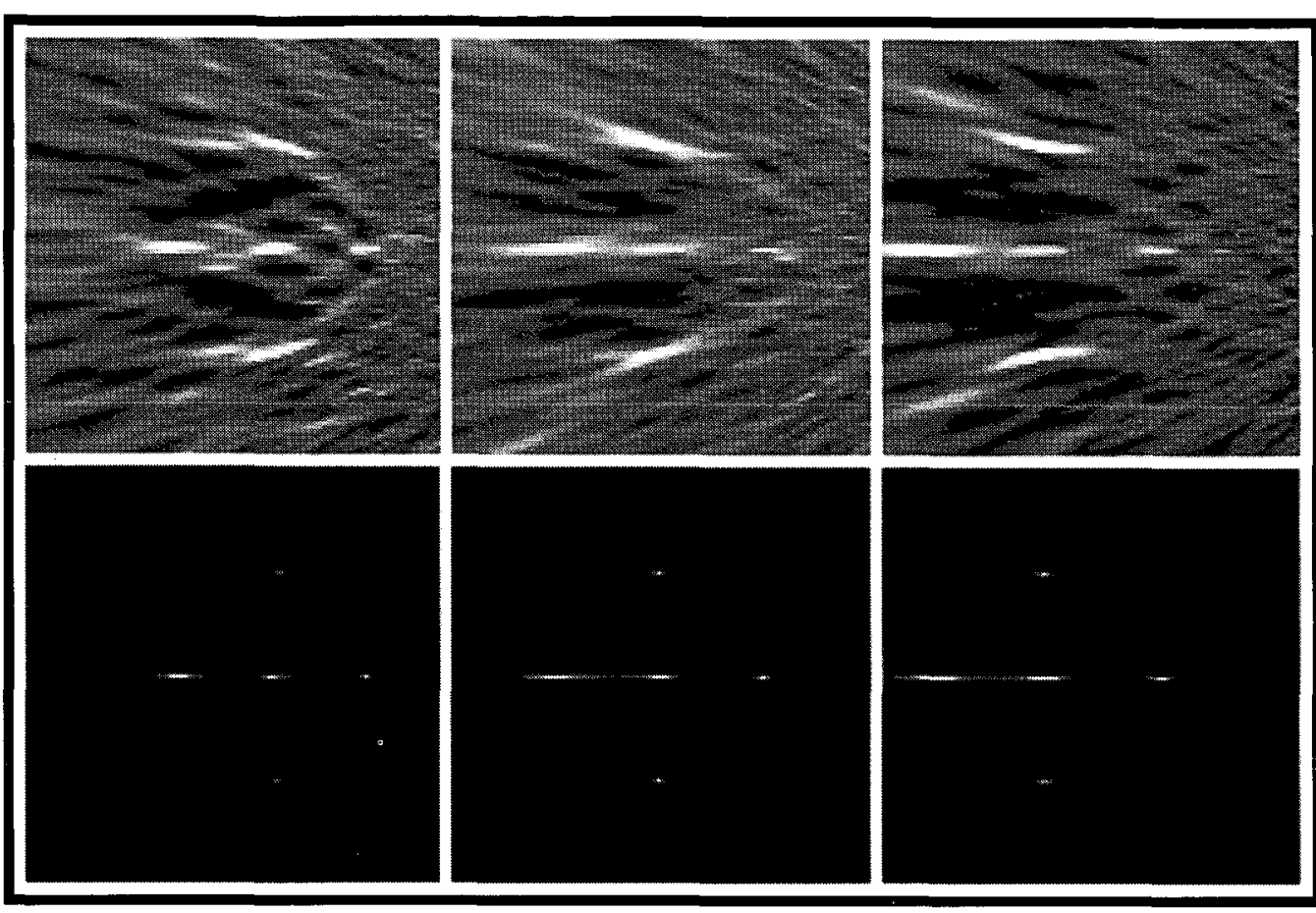

Beamforming

$10 \%$ slower

Correct

$10 \%$ faster

Velocity in medium

\section{Geometry}

Sources: 5 in '+' configuration Receivers: Linear array of 12 Receiver spacing: $50 \mathrm{ft}$.

Receiver range: $400 \mathrm{ft}$. from trmt. well Grid size: $500 \mathrm{ft}$. $x 500 \mathrm{ft}$. Grid spacing: $5 \mathrm{ft}$. $\times 5 \mathrm{ft}$.

\section{Reconstruction Parameters}

Reconstruction method: Beamforming \& MVDR Propagation model: Uniform Geometric attenuation: 1/r

Intrinsic attenuation: None

Amplitude compensation: None Display: Magnitude

\section{Signal Parameters}

Tube noise: $2 \mathrm{X}$

Uncorrelated sensor noise: $0.1 \mathrm{X}$ Source amplitude variation: None Velocity: $2200 \mathrm{ft} . / \mathrm{sec}$. (S-wave)

Frequency: $75 \mathrm{~Hz}$ to $200 \mathrm{~Hz}(6)$

Time-bandwidth product: 30

Figure 11 Constant velocity errors bias the reconstructed source locations using either the Beamforming or MVDR methods. The five-source pattern is relatively undistorted with constant velocity error, but shifts position. The reconstruction velocity was held constant and the actual propagation velocity was varied. 
sen too high, the interpretation must be that the source is closer to the array than it actually is. For low reconstruction velocities, the opposite is true.

These are the first reconstructions we show with the MVDR method. This method produces images with very high apparent resolution, and with considerable reduction in artifact clutter. Our experience is that the method actually doubles resolution, and effectively reduces processing sidelobes.

\subsubsection{More complicated media}

Matched field processing gives us the opportunity to image emitters in more complicated media, provided, again, that the velocity structure is known. To simulate propagation in a heterogeneous medium, we use a paraxial split-step algorithm [Thomson and Chapman, 1983]. The results of wave propagation simulation with this algorithm are illustrated in figure 12. To simplify, computation of the phase and amplitude of the Green's function at each of the 10,000 candidate source locations that are characteristic of our examples, we exploit the reciprocity theorem. Instead of solving the wave equation for each source position, we use reciprocity to swap the source and the receiver locations. The Green's functions are identical, in this case, but the wave equation need be solved only 12 times.

The monochromatic wave fields for two receiver (now source) positions are shown in figure 12 . The medium contains a velocity discontinuity: above the interface, the velocity is $5000 \mathrm{ft} / \mathrm{sec}$.; below, it is $6000 \mathrm{ft} / \mathrm{sec}$. The source on the left is above the interface in the lower velocity medium. An interference pattern to the left half of the figure results from a reflection off of the interface. The source in the right half of the figure is below the interface. The reflection coefficient is smaller in this case, so that an interference pattern is not observed. The wavelength of the field decreases as it refracts into the upper region, a result of the lower velocity in that region.

Figure 13 shows the result of matched field processing reconstructions using computed wavefields. The lower two images are the results of propagation in the step velocity medium of figure 12. On the bottom left the reconstruction is made using the correct step velocity wavefield. On the bottom right is the result of an incorrect reconstruction velocity. The reconstruction in this case was made with velocity that was $10 \%$ too high below the interface, and $10 \%$ too low above it. The image is badly distorted in this case, and it could not be said that the correct number of emitters or that the correct emitter locations are obtained. However, there is energy in the neighborhood of the emitters.

The top two images correspond to a different velocity structure: a velocity gradient. The velocity varied in this case from $4750 \mathrm{ft} / \mathrm{sec}$ at the top of the image to $5250 \mathrm{ft} / \mathrm{sec}$ at the bottom. Again, the image on the left was constructed using the correct velocity structure. The image on the right resulted from reconstruction with a uniform velocity of $5000 \mathrm{ft} / \mathrm{sec}$. In this case, the reconstruction with an erroneous velocity estimate is good. The number of sources apparent is correct, and their positions have not deviated too much from the correct locations. This image is distorted, but not too severely. 

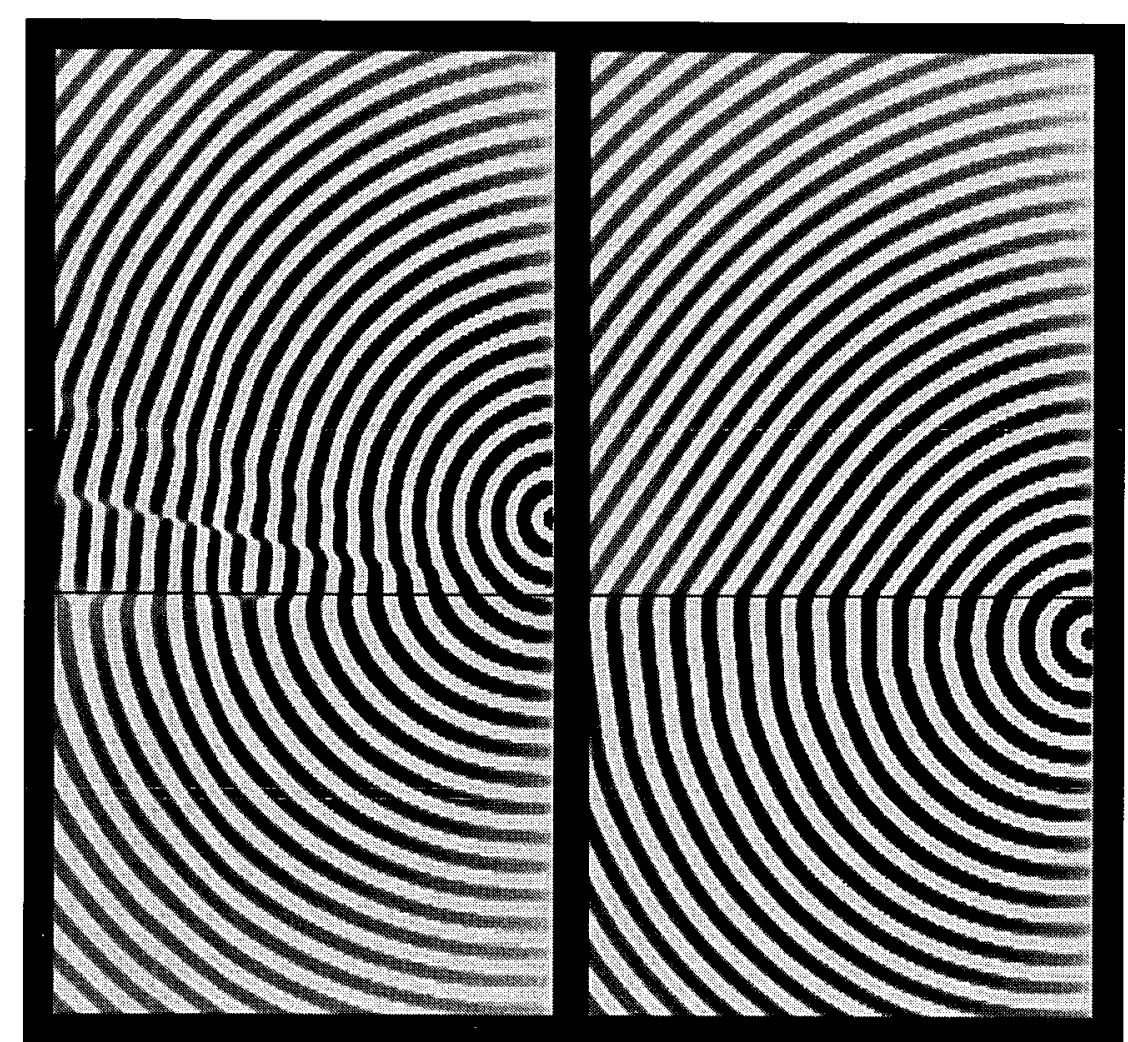

$5000 \mathrm{ft} . / \mathrm{sec}$.

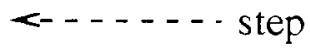

Receiver 6

Receiver 9

Geometry

Grid size: $500 \mathrm{ft}$ x $500 \mathrm{ft}$

Grid spacing: $2.5 \mathrm{ft}$. $x 5 \mathrm{ft}$.
Field Parameters

Geometric attenuation: $1 /\left(\mathrm{r}^{* *} 0.5\right)$
Intrinsic attenuation: Yes Intrinsic attenuation: Yes
Field Parameters

Velocity: As shown above Frequency: $100 \mathrm{~Hz}$

Figure 12 Matched Field Processing allows us to model propagation in complex media. Here we model propagation in a medium which has a step in propagation velocity from $5000 \mathrm{ft} / \mathrm{sec}$. to $6000 \mathrm{ft}$./sec. Receiver 6 is above the velocity step, and Receiver 9 is below the velocity step. Note the interference patterns due to a reflection in the Receiver 6 field. 


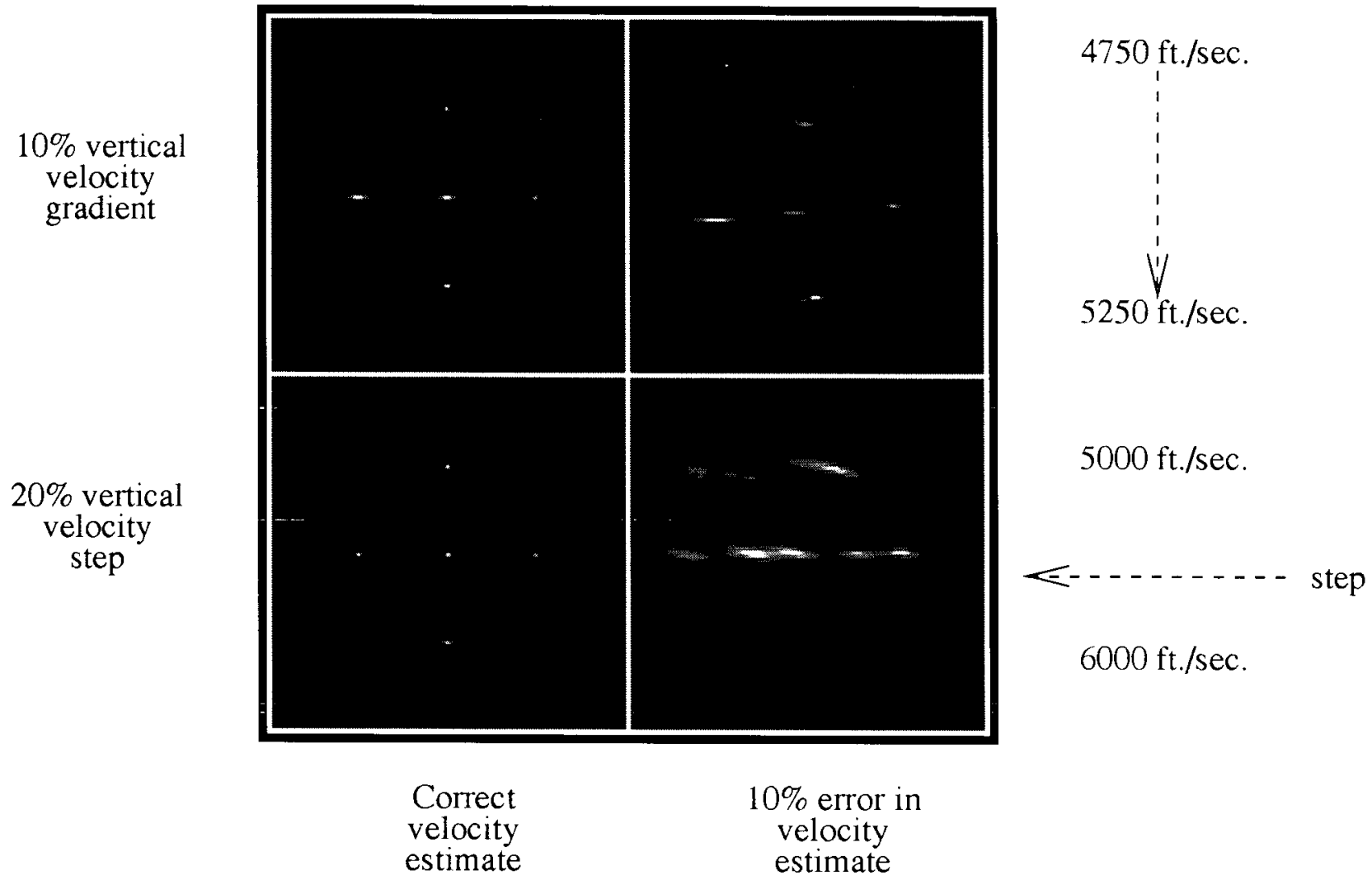

\section{Geometry}

Sources: 5 in 't' configuration

Receivers: Linear array of 12

Receiver spacing: $50 \mathrm{ft}$.

Receiver range: $400 \mathrm{ft}$. from trmt. well

Grid size $500 \mathrm{ft} \times 500 \mathrm{ft}$

Grid spacing: $2.5 \mathrm{ft} . \times 5 \mathrm{ft}$

\section{Reconstruction Parameters}

Reconstruction method: MVDR

Propagation model: Gradient or step Geometric attenuation: $1 /(\mathrm{r} * * 0.5)$

Intrinsic attenuation: Yes

Amplitude compensation: None

Display: Magnitude

\section{Signal Parameters}

Tube noise: None

Uncorrelated sensor noise: $0.01 \mathrm{X}$ Source amplitude variation: None

Velocity: As shown above

Frequency: $100 \mathrm{~Hz}$

Time-bandwidth product: 30

Figure 13 A ten percent error in the velocity estimate in a non-uniform medium may result in acceptable reconstructions. In the upper right image, simulated signals propagating through a linear velocity gradient (4750 to $5250 \mathrm{ft} . / \mathrm{sec}$.) are reconstructed assuming a uniform velocity medium (5000 ft./sec.). In the lower right image, simulated signals propagating in the step-velocity medium of figure 12 are reconstructed assuming a step medium having velocities of 4500 and $6600 \mathrm{ft} . / \mathrm{sec}$. 
One conclusion we draw from these examples is that the estimation of average medium velocity should be possible with matched field processing when perforation shots are available for calibration. The reconstruction velocity can be adjusted until images of the calibration shots are in the correct location. We also infer that $10 \%$ velocity errors may be tolerable when the errors do not include step discontinuities.

\subsection{Amplitude Compensation}

In our last two examples, we examine our concept for amplitude compensation. We use the five source cross test pattern of previous figures, but vary the amplitudes of the waveforms incident upon the array over an order of magnitude. The signals from one source (at the center of the pattern) have uniform amplitude across the array. The other four sources produce signals that vary in amplitude from 0.1 to 1.0 over the array aperture. The resulting single-frequency and frequency-averaged MVDR reconstructions for varying signalto-noise ratio are shown in figure 14 .

Amplitude compensation makes it possible to see the sources with strong amplitude anomalies, but introduces significant artifacts. Not all of the artifacts are suppressed by frequency averaging. Those that remain may be due to an ambiguity between phase and amplitude corrections. A phase error of $180^{\circ}$ can be compensated by an amplitude correction of -1 . Consequently, ghost events may occur in those areas where many or most of the measured array signals are 180 degrees out of phase with respect to the vector of Green's functions. Ghost images appear in our reconstructions between legitimate sources.

We also studied the effects of varying SNR, in this case using spatially uncorrelated noise. Amplitude compensation provides some protection from varying amplitude at high SNR, but degrades significantly at low SNR (0.1). We surmise that the increase in the number of degrees of freedom permits the model to match the noise, resulting in the signal being overwhelmed by artifacts. At low SNR when the noise is spatially uncorrelated, the MVDR method approaches conventional beamforming, which is more robust to amplitude error, but has less resolution.

We hypothesized that the artifacts were exacerbated by the fact that the array was undersampled. To examine this possibility, we simulated reconstructions with an array of 48 sensors (figure 15 ). The array aperture was unchanged $(550 \mathrm{ft}$ ), but the inter-element spacing was reduced to $12.5 \mathrm{ft}$ (from $50 \mathrm{ft}$ ). Both conventional beamforming and MVDR reconstructions were attempted with and without amplitude compensation at an SNR of 1.0. The artifacts are still present, even with frequency averaging. Amplitude compensation appears to degrade images produced by beamforming. Beamforming without compensation appears to be fairly robust to amplitude errors, although resolution is poor. The MVDR method appears to be more sensitive to amplitude error, as might be expected of a high-resolution method. Compensation definitely improved the MVDR image, making all sources visible at the cost of increased artifacts.

One notable effect of increasing the number of sensors was to suppress the noise more effectively. The 48-element array produced an image at an SNR of 1.0 comparable to the 12 element array at an SNR of 10.0 . 


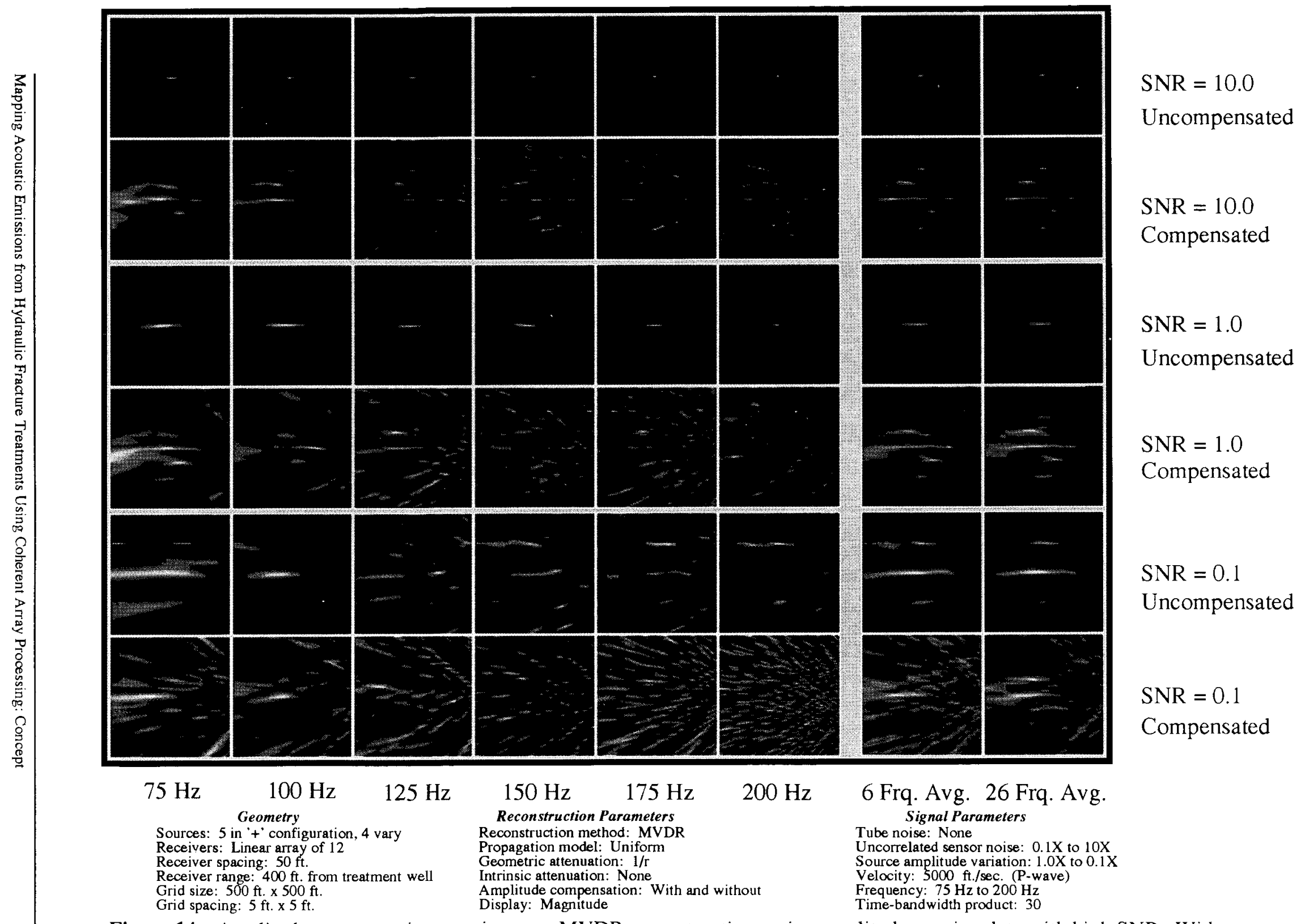

Figure 14 Amplitude compensation may improve MVDR reconstructions using amplitude-varying data with high SNR. Without compensation, some sources are suppressed. Compensation equalizes source reconstructions, but introduces artifacts. 


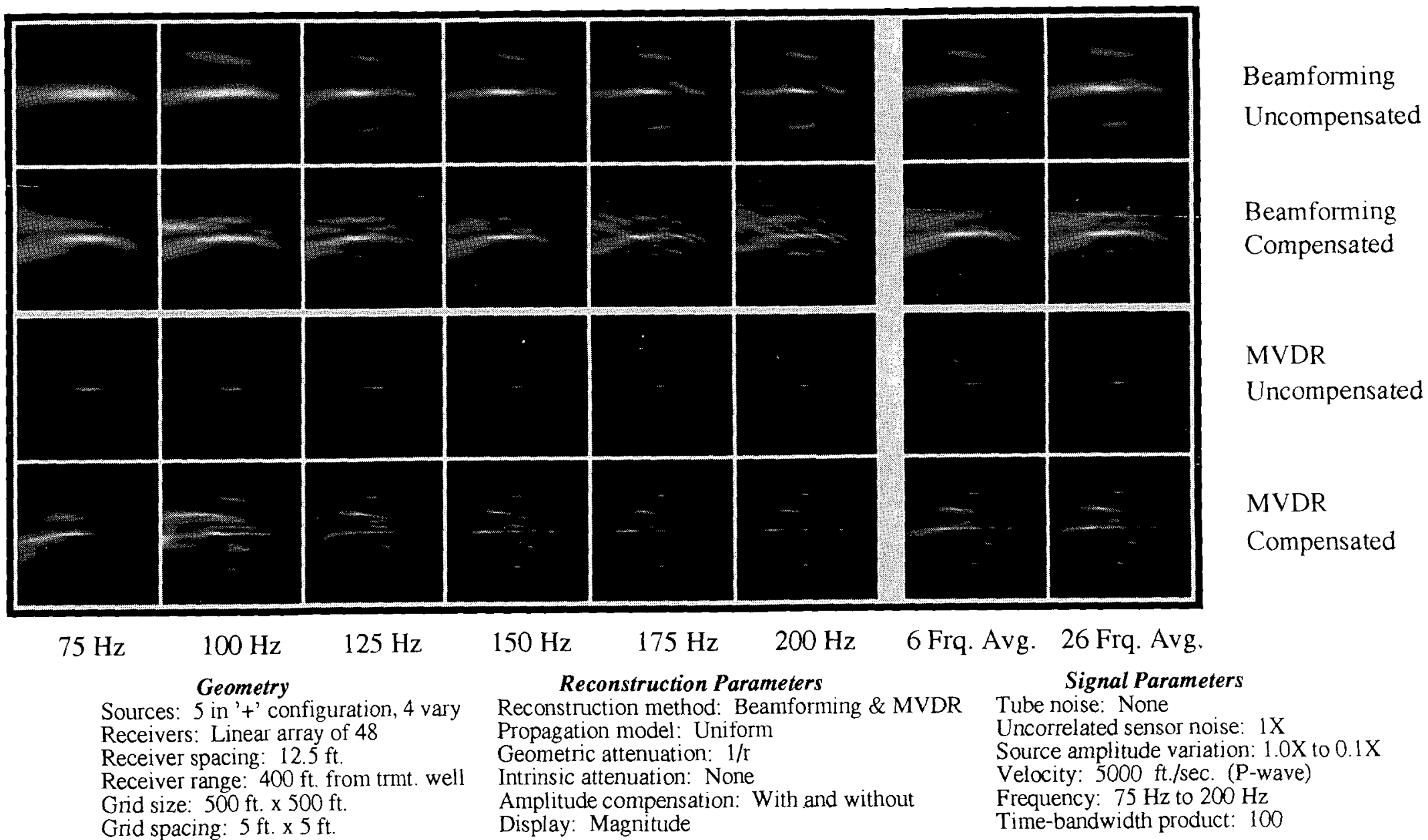

Figure 15 Reducing the receiver spacing while maintaining the length of the receiver array improves the reconstruction of amplitude-varying data. The receiver array here consists of 48 receivers at a spacing of 12.5 feet (as opposed to 12 receivers at a spacing of 50.0 feet for figure 14). Reconstruction quality at $S N R=1$ here is comparable to SNR=10 in figure 14. 
The denser array also eliminate problems with aliasing. Comparing the second row of images in figure 14 with the fourth row in figure 15 , we see a substantial reduction in the number of artifacts. Most of the artifacts are suppressed for the twelve element array by frequency averaging. Those artifacts eliminated by frequency averaging in the smaller array are not present for even a single frequency in the denser array. Thus, there are two paths to the elimination of aliasing errors: increasing the number of sensors, or increasing the processing bandwidth.

We conclude that amplitude compensation is not warranted except at high SNR $(>1.0)$. Amplitude compensation may be improved by constraining the elements of the amplitude correction vector to be positive. This constraint may eliminate ghosts due to $180^{\circ}$ phase ambiguities.

\subsection{Conclusions}

Coherent processing methods appear to have sufficient resolution in the 75 to $200 \mathrm{~Hz}$ band to delineate the extent of fractures induced by hydraulic fracturing. The medium velocity structure must be known with a $10 \%$ accuracy or better and no major discontinuities should go undetected. For best results, the receiving array must be positioned directly opposite the perforations (same depth) at a horizontal range of 200 to 400 feet from the region to be imaged. With the MVDR method and frequency-averaging, sources of acoustic emission may be detectable down to a single-sensor SNR of 0.25 or somewhat less.

These conclusions are limited by the assumptions of this study: good coupling to the formation, acoustic propagation, and accurate knowledge of the velocity structure. We indicate now how the last two of these restrictions might be relaxed.

\subsection{Extensions to Elastic Propagation}

Propagation may consist of both compressional and shear modes. Although matched field processing has been presented in this report for the acoustic case, there is no fundamental impediment to matched field processing for elastic propagation. The simplest approach is to substitute a numerical elastic propagation simulator for the acoustic simulator used to compute narrowband Green's functions. A suitable finite difference method is given by Pratt[1990].

Under conditions of weak velocity variations, compressional and shear fields might decouple. In this case it may be possible to use an acoustic simulator twice to generate a matching elastic field, operating once each for compressional and shear velocity models. The principal limitation of this approach would be that no P-S or S-P conversions could be modelled. The elastic field will be a sum of compressional $\varepsilon_{P}$ and shear $\underline{\varepsilon}_{S}$ fields, with unknown complex weights $\alpha_{P}$ and $\alpha_{S}$.

For the conventional beamforming method, the beam energy of equation 13 would have to be modified to account for the sum of compressional and shear fields: 


$$
E=\left(\alpha_{P} \varepsilon_{P}+\alpha_{S} \varepsilon_{S}\right)^{H} R\left(\alpha_{P} \varepsilon_{P}+\alpha_{S} \varepsilon_{S}\right)
$$

Here, the steering vectors are assumed to have been normalized. The relative compressional and shear excitations are unknown, but can be estimated by maximizing the energy with respect to the excitation weights. The expression (equation 33) for the beam energy can be simplified using the form:

$$
E=\underline{\alpha}^{T} \underline{\Gamma}^{H} \underline{R} \underline{\underline{\alpha}} \quad \underline{\Gamma}=\left[\underline{\varepsilon}_{P} \underline{\varepsilon}_{S}\right] \quad \underline{\alpha}=\left[\begin{array}{l}
\alpha_{p} \\
\alpha_{S}
\end{array}\right]
$$

As with the amplitude compensation strategy, we propose to maximize this quantity subject to the constraint $\underline{\alpha}^{H} \underline{\alpha}=1$, leading to an eigenvalue problem. In this case it is a complex eigenvalue problem with the solution:

$$
E=\lambda_{\max }\left(\underline{\Gamma}^{H} \underline{R} \underline{\Gamma}\right)
$$

The compressional and shear wave weights are components of the eigenvector corresponding to the largest eigenvalue.

In a similar fashion, the MVDR method can be modified to use this acoustic-elastic strategy. Equation 32 is modified as:

$$
E=\frac{1}{\left(\alpha_{P} \varepsilon_{P}+\alpha_{S} \varepsilon_{S}\right)^{H} \underline{R}^{-1}\left(\alpha_{P} \varepsilon_{P}+\alpha_{S} \varepsilon_{S}\right)}
$$

and the corresponding maximum matched field value is given by:

$$
E=\lambda_{\min }^{-1}\left(\underline{\Gamma}^{H} \underline{R} \underline{\Gamma}\right)
$$

In this case, the compressional and shear weights are elements of the eigenvector corresponding to the minimum eigenvalue.

This method is potentially fast because it allows us to calculate compressional and shear fields separately with a paraxial acoustic propagation scheme. These are substantially faster than finite difference methods for solving the elastic boundary value problem.

\subsection{Calibration and Tomography}

Perforation shots potentially provide calibration information on the propagation medium between the treatment and measurement wells. Matched field processing can be used as the inner loop of an iterative scheme to estimate the medium velocity structure. This approach has been proposed for ocean acoustic tomography [Tolstoy and Diachok, 1991]. The general idea is to maximize the matched field energy at the known locations of perfo- 
ration sources by adjusting (a hopefully few) parameters describing the velocity structure. For constant velocity media, our simulations of matched field images for constant velocity errors suggests that this form of tomography will work. The images obtained had the correct pattern, but were simply shifted from their correct locations. Adjusting the velocity in this case should be a simple matter of aligning the sources to their proper locations.

\subsection{Acknowledgements}

The authors would like to thank Dr. Stan Trost for funding this research under the New Initiatives thrust area. The authors would also like to thank Dr. John Fairborn of Chevron Oil Field Research Company for many helpful suggestions, advice, and for providing an opportunity to acquire data to test the ideas proposed in this document. The authors would also like to thank Dr. Arthur Lewis for his support.

Work performed under the auspices of the U. S. Department of Energy by the Lawrence Livermore National Laboratory under Contract W-7405-Eng-48.

\subsection{References}

Acharya, R. (1988), "Hydraulic-fracture-treatment design simulation," Jour. of Pet. Tech., (Feb. 1988), 139-142.

Ahmed, U. (1988), "Fracture height prediction," Jour. of Pet. Tech., (July 1988), 813-815.

Anderson, R., and A. Phillips, (1988), "Practical application of economic well-performance criteria to the optimization of fracturing treatment," Jour. of Pet. Tech., (Feb. 1988), 223-228.

Albright, J. and R. Hanold (1983), "Acoustic emissions as a tool for hydraulic fracture locations: experience at the Fenton Hill hot dry rock site," Soc. Petro. Engrs J., 523-530.

Baggeroer, A., W. Kuperman and H. Schmidt (1988), "Matched field processing: source localization in correlated noise as an optimum parameter estimation problem, J. Acoust. Soc. Am., 83(2), 571-587.

Chevron World (1991), "Frac attack," Chevron World, 68(2), 24-27.

Chouzenoux, C. (1991), "Measurement of borehole seismic noise with hydrophones," Geophysics, 56(3), 1437-1444.

Cleary, M. (1988), "The Engineering of Hydraulic Fractures - State of the Art and Technology of the Future," Jour. of Pet. Tech., Jan. 1988, 13-21.

Economides, M. (1987), "How to engineer a fracturing treatment," Jour. of Pet. Tech., (Nov. 1987), 1343-1345. 
Elbel, J. (1988), "Considerations for optimum fracture geometry design," SPE Prod. Eng., (Aug. 1988), 323-327.

Engi, D. (1987), "A numerical signal processing technique for borehole fracture diagnostics," Sandia report SAND86-1865, Sandia Natl. Lab.,Albuquerque NM (Jan. 1987).

Fehler, M. (1989), "Stress Constrol of Seismicity Patterns Observed during Hydraulic Fracturing Experiments at the Fenton Hill Hot Dry Rock Geothermal Energy Site, New Mexico," Int. J. Rock Mech. Min. Sci. \& Geomech. Abstr., 26(3/4), 221-219.

Fehler, M., L. House and H. Kaieda (1987), "Determining planes along which earthquakes occur: method and application to earthquakes accompanying hydraulic fracturing," J. Geophys Res., 92, 9407-9414.

Fix, J., R. Adair, T. Fisher, K. Mahrer, C. Mulcahy, B. Myers, J. Swanson, J. Woerpel, ( 1989) "Development of microseismic methods to determine hydraulic fracture dimensions," Tech. Report no. 89-0116, Teledyne Geotech, Garland, TX.

Harris, D., S. Jarpe, P. Harben (1991), "Seismic noise cancellation in a geothermal field," to appear Geophysics, 56(10), UCRL- , Lawrence Livermore National Laboratory, Livermore, $\mathrm{CA}$.

Hunt, E. (1991), "Frac height may increase away from well bore," Oil and Gas Jour., (Feb. 25, 1991), 33-36.

Knight, W., R. Pridham, and S. Kay (1981), Digital signal processing for sonar, Proc. IEEE, 69(11), 1451-1506.

Marzetta, T., M. Orton, A. Krampe, L. Johnston, and P. Wuenschel (1988), "A hydrophone vertical seismic profiling experiment," Geophysics, 53(11), 1437-1444.

Mauk, F. and K. Mahrer (1988), "Use of long-period seismometers to determine induced fracture geometry," SPE Prod. Eng., (May 1988), 192-200.

Power, D. (1977), "Acoustic emissions following hydraulic fracturing in a gas well," Proc. first conf. on acoustic emissions/microseismic activity in geologic structures and materials, Trans Tech Publications, CH-4711 Aedermannsdorf, Switzerland, 291-308.

Pratt, R. (1990), "Frequency-domain elastic wave modeling by finite differences: A tool for crosshole seismic imaging," Geophysics, 55(5), 626-632.

Shuck, L. and T. Keech (1977), "Monitoring acoustic emission from propagating fractures in petroleum reservoir rocks," Proc. first conf. on acoustic emissions/microseismic activity in geologic structures and materials, Trans Tech Publications, CH-4711 Aedermannsdorf, Switzerland, 309-338.

Thomson, D. and N. Chapman (1983), "A wide-angle split-step algorithm for the parabolic equation," Jour. of the Acoustic Soc. of Am., 74(6), 1848-1854. 
Thorne, B. and H. Morris (1988a), "Passive seismic monitoring of hydraulic fracture experiments at the multiwell experiment site," Sandia report SAND88-1284, Sandia Natl. Lab., Albuquerque, NM (June 1988).

Thorne, B. and H. Morris (1988b), "Advances in borehole seismic fracture diagnostics," SPEFE (Dec. 1988), 711-715.

Tolstoy, A. and O. Diachok (1991), "Acoustic tomography via matched field processing," Jour. of the Acoustical Soc. of Am., 89(3), 1119-1127.

Veatch, R. (1983a), "Overview of current hydraulic fracturing design and treatment technology - part 1," Jour. of Pet. Tech., (April 1983), 677-687.

Veatch, R. (1983b), "Overview of current hydraulic fracturing design and treatment technology - part 2," Jour. of Pet. Tech., (May 1983), 853-864.

Waters, B. (1981), "Hydraulic fracturing - what is it?," Jour. of Pet. Tech., (Aug. 1981), 1416. 